\title{
An Analysis of the Saturation of a High Gain FEL*
}

\author{
Robert L. Gluckstern \\ Department of Physics \\ University of Maryland \\ College Park, MD 20742 \\ Samuel Krinsky \\ National Synchrotron Light Source \\ Brookhaven National Laboratory \\ Upton, NY 11973 \\ and \\ Hiromi Okamoto ${ }^{\dagger}$ \\ Department of Physics \\ University of Maryland \\ College Park, MD 20742
}

December 14, 1992

\begin{abstract}
We study the saturated state of an untapered free electron laser in the Compton regime, arising after exponential amplification of an initial low level of radiation by an initially monoenergetic, unhunched electron beam. The saturated state of the FEL is described by oscillations about an equililbrium state.
\end{abstract}

-Work performed under the auspices of the U.S. Department of Energy

†Permanent address, Institute for Chemical Research, Kyoto University, Kyoto 611, Japan 
Using the two invariants of the motion, and certain assumptions motivated by computer simulations, we provide approximate analytic descriptions of the radiation field and electron distribution in the saturation regime. We first consider a one-dimensional approximation, and later extend our approach to treat an electron beam of finite radial extent. Of note is a result on the radiated power in the case of an electron beam with small radius.

\section{Introduction}

In this paper we study the saturated state of an untapered free electron laser in the Compton regime. Guided by the results of simulations starting with a monoenergetic unbunched electron beam and a low initial level of radiation, we make assumptions which prove to give an accurate picture of what happens in the saturation regime. The solutions in the saturated regime are related to the initial conditions by using the two invariants of the motion. We first consider a one-dimensional approximation, and later extend our approach to treat an electron beam of finite radial extent, including the effects of the diffraction of the radiation and the radiation focusing properties of the electron beam bunched by the FEL interaction.

The starting point of the analysis is the scaled equations for the evolution of the one dimensional electron distribution and for the monochromatic radiation field. The notation is that of Bonifacio et. al. ${ }^{1}$ and the equations are

$$
\begin{gathered}
\frac{d \sigma_{j}}{d \tau}=p_{j}, \\
\frac{d p_{j}}{d \tau}=-A e^{i \sigma,}-A^{*} e^{-i \sigma_{j}}, \\
\frac{d A}{d \tau}=<e^{-i \sigma,}>+i A \delta,
\end{gathered}
$$

where $\sigma_{j}$ and $p_{j}$ are the phase of the jth electron relative to the radiation and its (scaled) momentum deviation, $A$ is the (scaled) amplitude of the radiation at the (scaled) longitudinal position $\tau=2 \rho k_{w} z$, whire $2 \pi / k_{w}$ is the wiggler period and $\rho$ is the Pierce parameter, $\delta$ is the detuning of the laser, and $<>$ is an average over the electron distribution.

It is easy to show from Eqs. (1.1)-(1.3) that

$$
<p_{j}>+|A|^{2}=C_{1}
$$


and

$$
\frac{<p_{j}^{2}>}{2}+2 \operatorname{Im}\left[A<e^{i \sigma_{j}}>\right]-\delta|A|^{2}=C_{2}
$$

are constants of tive motion. For an initially monoenergetic unbunched electron beam and a low initial lev ol of radiation, the constants $C_{1}$ and $C_{2}$ are approximated by zero.

In Fig. 1.1 we show a typical evolution of the radiation with $\tau$. The field builds up exponentially as the electrons bunch. After the bunched electrons are captured in buckets, the radiation oscillates with modest amplitude about an equilibrium distribution. In Fig. 1.2 sve show the phase of the radiation as a function of $\tau$. It appears that this phase is very nearly linear with $\tau$. We shall take advantage of this behavior and recast our equations by writing

$$
A=(P+i Q) e^{i \nu(\tau-\tau)}
$$

where $\nu$ and $\tau_{0}$ are chosen to correspond to the average slope and intercept in Fig. 1.2. In fact we will later predict (Section 3.2) the value of $\nu$, and it will agree closely with the value appropriate to Fig. 1.2.

The saturated state of the FEL is described by oscillations about an equilibrium state. $^{2-5}$ This equilibrium state corresponds to a steady state solution of Eqs. (1.1)(1.3). The proper choice of the equilibrium solution is significantly restricted 2 by the two invariants of Eqs. (1.4) and (1.5), relating properties of the saturated state back to the initial conditions at the start-up of the FEL. In Section 3, we study the properties of the equilibrium solution. The equilibrium radiation field has the form

$$
A=P_{0} e^{i \nu\left(r-r_{0}\right)}
$$

where $P_{0}$ is constant. We introduce the displaced electron phase $\phi_{j}(\tau)$ in the equilibrium state

$$
\phi_{j} \equiv \sigma_{j}+\nu\left(\tau-\tau_{0}\right)+\frac{\pi}{2},
$$

and require $\nu$ to be chosen such that

$$
<\phi_{j}^{\prime}>=0
$$

In the case of zero detuning, $\delta=0$, we find

$$
<\sin \phi_{j}>=0
$$




$$
\begin{gathered}
<\cos \phi_{j}>=P_{0}^{3}, \\
<\phi_{j}^{22}>=3 P_{0}^{4}, \\
\nu=P_{0}^{2} .
\end{gathered}
$$

The equilibrium electron distribution has the form

$$
f\left(\phi, \phi^{\prime}\right)=F(H)
$$

where

$$
H=\phi^{\prime 2} / 2-2 P_{0} \cos \phi .
$$

We consider three quite different choices for $F(H)$ :

1. $F_{K V}(H)=N_{1} \delta\left(H-H_{0}\right) \quad$ (KV-distribution)

2. $F_{-1 / 2}(H)=N_{2}\left(H_{0}-H\right)^{-1 / 2}$

3. $F_{B}(H)=N_{3} \exp (-\alpha H) \quad$ (Boltzmann distribution)

Suprisingly, we find that in all three cases, $P_{0} \cong 0.81$, in good agreement with Fig. 1.1. Moreover, from computer simulation results, it appears that the actual electron distribution arising after the saturation of the initial exponential growth is similar to the Boltzmann distribution.

A simplified model of oscillations about the equilibrium state is presented in Section 4 , based on an ansatz retaining only a single harmonic:

$$
\begin{gathered}
P(\tau)=P_{0}+P_{1} \cos \Omega \tau, \\
Q(\tau)=Q_{1} \sin \Omega \tau, \\
\beta_{j}(\tau) \equiv \sigma_{j}(\tau)+\nu\left(\tau-\tau_{0}\right)+\frac{\pi}{2}=\phi_{j}(\tau)+a \sin \Omega \tau .
\end{gathered}
$$

A more exact treatment is given in Section 5 , using the Vlasov equation. The coherent frequency $\Omega$ is determined and shown to agree with computer simulation.

The work presented in this paper carries forward that of Lane and Davidson ${ }^{2}$, who used the invariants to constrain the equilibrium solutions, relating them to the initial conditions at start-up. The equations we use are equivalent to those employed by Sharp and Yu 4,5 in their study of the sideband instability; however, in our case 
we consider a radiation field depending on axial coordinate $z$, but independent of time $t$. Sharp and $\mathrm{Yu}$ do not use the invariants to restrict the equilibrium solutions. In this paper we provide explicit numerical comparisons between our analytical work and computer simulations, finding good agreement.

The paper is organized as follows: In Section 2 we review the derivation of the growth rate of the radiation in the exponential regime. In Section 3 we obtain the differential equations for $P$ and $Q$ as functions of $\tau$, and discuss the formulation of the equilibrium distribution in the saturation regime. In Section 4 we solve these equations for $P$ and $Q$ as functions of $\tau$ by assuming that the oscillations about equilibrium are dominated by a first harmonic. In Section 5 we use the Vlasov equation to explore the coherent frequency of small oscillations about equilibrium, and discuss the stability of these oscillations. In Section 6 we utilize numerical simulations to further study the stability of these oscillations. Theory and simulation both show that for the case of interest, the small amplitude oscillations are unstable. The moderate amplitude oscillations observed in the saturated state presumably correspond to saturation of this instability at large enough amplitude. In Section 7 we examine the effects of detuning and energy spread. In Section 8 we explore the consequences of using an electron beam with a finite radial extent. Section 9 is then a summary of the main conclusions of this paper.

\section{Exponential Growth Regime ${ }^{6}$}

We start with a low level of radiation $(|A| \ll 1)$ and an approximately uniform distribution in electron phase, $\sigma_{j}$. Taking two derivatives of Eq. (1.3) leads to

$$
\frac{d^{3} A}{d \tau^{3}}-i \delta \frac{d^{2} A}{d \tau^{2}}=-i<\left(\frac{d^{2} \sigma_{j}}{d \tau^{2}}-i\left(\frac{d \sigma_{j}}{d \tau}\right)^{2}\right) e^{-i \sigma,}>
$$

Since we are here interested only in terms linear in $p_{j}$ and $A$, we drop the quadratic term involving $\left(d \sigma_{j} / d \tau\right)^{2}$ and use Eqs. (1.1) and (1.2) to obtain.

$$
\frac{d^{3} A}{d \tau^{3}}-i \delta \frac{d^{2} A}{d \tau^{2}}-i . A=i A^{*}<e^{-2 i \sigma}>
$$

Since the right side of Eq. (2.2) is quadratic in $A$ if we start with a distribution in electron phase which is approximately uniform, we obtain the linear equation for $A$ :

$$
\frac{d^{3} A}{d \tau^{3}}-i \delta \frac{d^{2} A}{d \tau^{2}}-i A=0
$$


The general solution of Eq. (2.3) is

$$
A=e^{i \mu \tau},
$$

where

$$
\mu^{3}-\delta \mu^{2}+1=0 .
$$

The exponential growth solution corresponds to the complex root for $\mu$ with a negative imaginary part. For zero detuning $(\delta=0)$ this is

$$
\mu=\frac{1}{2}-i \frac{\sqrt{3}}{2}
$$

corresponding to the exponential growth

$$
A \simeq A_{o} e^{\left(\frac{\sqrt{3}}{2}+\frac{i}{2}\right) \tau} .
$$

For small non-zero detuning $(\delta<1)$, the growth in the radiation amplitude is approximately

$$
A \simeq A_{\circ} e^{\frac{\sqrt{3}}{2}\left(1-\frac{\sigma^{2}}{3}\right) \tau+i\left(\frac{1}{2}+\frac{\delta}{3}+\frac{\sigma^{2}}{18}\right) \tau} .
$$

Equation (2.7) (or its generalization in Eq. $(2.8)$ for $\delta \neq 0$ ) is expected to govern the evolution of Eqs. (1.1)-(1.3) until $|A(\tau)|$ becomes of order 1, when our assumptions are no longer appropriate and some form of saturation will take place.

\section{Saturation Regime}

\subsection{Behavior of the Radiation Phase}

Numerical simulations ${ }^{7}$ of Eqs. (1.1)-(1.3) starting with a monoenergetic uniform electron phase distribution and a low radiation level indicate that the phase of the complex radiation amplitude $A$ is very nearly linear with $\tau$. A typical result is shown in Fig. 1.2 for the phase of the radiation as a function of $\tau$ for a starting radiation level corresponding to $|A|=.01$. In Fig. 1.1 we show the corresponding field amplitude $|A|$ as a function of $\tau$ which clearly exhibits the early exponential growth as well as the transition to saturation when $|A|$ is of order 1 . And in Fig. 3.1 we show the phase space distribution of the electrons, which initially were monoenergetic with a uniform phase distribution, in the saturation regime at $\tau \sim 9$. 
It is clear that the electrons have been bunched during the buildup of the radiation, and are now oscillating with fairly large amplitudes. The oscillation of the radiation shown in Fig. 1.1 is also of large amplitude $( \pm 50 \%)$. But there is a clear indication that some sort of steady state has been reached in the saturation regime. In fact, simulations show that the steady state configuration is essentially independent of the initial electron and radiation configuration, provided we start with an approximately monoenergetic unbunched electron beam, and a low level of radiation.

We now rewrite Eqs. (1.1)-(1.5) in terms of $P(\tau), Q(\tau)$ and the electron phase $\beta_{j}$ given by

$$
\beta_{j}=\sigma_{j}+\nu\left(\tau-\tau_{0}\right)+\pi / 2
$$

In Fig. 3.2 we show $P(\tau)$ and $Q(\tau)$ from the simulation, and in Fig. 3.3 show the phase space distribuition in the $\beta_{j}^{\prime}, \beta_{j}$ space at $\tau=10,15,20,25,30,35$, where $\beta_{j}^{\prime}=\sigma_{j}^{\prime}+\nu=p_{j}+\nu$. Here the prime stands for $d / d \tau$. It is clear that the electrons have formed a bunch which rotates and oscillates as $\tau$ increases. In Fig. 3.3, we plot only those electrons which appear to have been captured in the bucket. Also $P(\tau)$ appears to oscillate sinusoidally about an equilibrium value $P_{0}$, and $Q(\tau)$ oscillates about zero (when $\tau_{0}$ is chosen appropriately).

Equations (1.1), (1.2) and (1.3) can now be written as ${ }^{8}$

$$
\begin{gathered}
\beta_{j}^{\prime}=p_{j}+\nu \\
\beta_{j}^{\prime \prime}=-2 P \sin \beta_{j}-2 Q \cos \beta_{j}, \\
Q^{\prime}+(\nu-\delta) P=<\cos \beta_{j}> \\
P^{\prime}-(\nu-\delta) Q=<\sin \beta_{j}>
\end{gathered}
$$

and the two invariants in Eqs. (1.4) and (1.5) become

$$
\begin{gathered}
<\beta_{j}^{\prime}>+P^{2}+Q^{2}-\nu=C_{1}, \\
\frac{<\beta_{j}^{\prime 2}>}{2}-\nu<\beta_{j}^{\prime}>+\frac{\nu^{2}}{2}-2 P<\cos \beta_{j}>+2 Q<\sin \beta_{j}>-\delta\left(P^{2}+Q^{2}\right)=C_{2} .
\end{gathered}
$$

Even though we have plotted only those electrons trapped in the bucket in Fig. 3.3, we note that all averages must be taken over all electrons, including those not trapped. 


\subsection{Equilibrium Distribution ${ }^{2-5}$}

As a first approximation, we will assume that the amplitude of oscillation is small, and we will derive the properties of the equilibrium distribution, assuming $C_{1}=C_{2}=0$. Identifying $\phi_{j}$ as the equilibrium electron phase distribution, and writing $P=P_{0}$ (constant) and $Q=0$, we have

$$
\begin{gathered}
(\nu-\delta) P_{0}=<\cos \phi_{j}> \\
<\sin \phi_{j}>=0
\end{gathered}
$$

and from Eqs. (3.6) and (3.7), together with $\left\langle\phi_{j}^{\prime}\right\rangle=0$, we find

$$
\nu=P_{0}^{2},\left\langle\phi_{j}^{\prime 2}\right\rangle=4 P_{0}<\cos \phi_{j}>+2 \delta P_{0}^{2}-\nu^{2}=3 P_{0}^{4}-2 \delta P_{0}^{2} .
$$

For $P=P_{0}, Q=0$, we see from Eq. (3.3) that any function of

$$
H\left(\phi_{j}, \phi_{j}^{\prime}\right)=\phi_{j}^{\prime 2} / 2-2 P_{0} \cos \phi_{j}
$$

will be a stationary distribution. For the appropriately normalized KV distribution

$$
f_{K V}\left(\phi_{j}, \phi_{j}^{\prime}\right)=N \delta\left(2 P_{0} \cos \phi_{j}-\phi_{j}^{\prime^{2}} / 2-2 P_{0} \cos \theta_{0}\right)
$$

corresponding to pendula oscillations with the same maximum angle $\theta_{0}$ but different phases, we find

$$
[K V]<\cos \phi_{j}>=\frac{2 E(m)}{K(m)}-1, \frac{<\phi_{j}^{\prime 2}>}{2}=4 P_{0}\left(\frac{E(m)}{K(m)}-1+m\right),
$$

where $m=\sin ^{2}\left(\theta_{0} / 2\right)$ and where $K(m)$ and $E(m)$ are the complete elliptic integrals of the first and second kind. For the phase space distribution

$$
f_{-\frac{1}{2}}\left(\phi_{j}, \phi_{j}^{\prime}\right)=N\left(2 P_{0} \cos \phi_{j}-\phi_{j}^{\prime 2} / 2-2 P_{0} \cos \theta_{0}\right)^{-1 / 2}
$$

we find

$$
\left[\left(H_{0}-H\right)^{-1 / 2}\right] \quad<\cos \phi_{j}>=\frac{\sin \theta_{0}}{\theta_{0}}, \frac{<\phi_{j}^{2}>}{2}=P_{\prime}\left(\frac{\sin \theta_{0}}{\theta_{0}}-\cos \theta_{0}\right),
$$

and for the Boltzmann distribution

$$
f_{B}\left(\phi_{j}, \phi_{j}^{\prime}\right)=N \exp \left(-\alpha \phi_{j}^{\prime^{2}} / 2+2 \alpha P_{0} \cos \phi_{j}\right)
$$


we find

$$
\text { [Boltzmann] }<\cos \phi_{j}>=\frac{I_{1}\left(2 \alpha P_{0}\right)}{I_{0}\left(2 \alpha P_{0}\right)}, \frac{\left\langle\phi_{j}^{\prime 2}>\right.}{2}=\frac{1}{2 \alpha} .
$$

Combining Eqs. (3.8), (3.10) we have the requirements

$$
\begin{gathered}
<\cos \phi_{j}>=P_{0}\left(P_{0}^{2}-\delta\right), \\
<\phi_{j}^{r^{2}}>=3 P_{0}^{4}-2 \delta P_{0}^{2} .
\end{gathered}
$$

Thus the combination of Eqs. (3.18), (3.19) and either (3.13), (3.15) or (3.17) determine the value of $P_{0}$ and $\theta_{0}$ (or $\alpha$ ) for each distribution.

Let us now consider the case $\delta=0$, in which case we can write

$$
<\phi_{j}^{\prime 2}>=3 P_{0}<\cos \phi_{j}>\text {. }
$$

For the KV beam, this requires

$$
\frac{2 E(m)}{K(m)}=5-8 m
$$

which leads to

$$
[\mathrm{KV}] \quad m=.433, \theta_{0}=82.3^{\circ}, P_{0}=.813,<H>=-0.218
$$

For the $\left(H_{0}-H\right)^{-1 / 2}$ distribution we find $\tan \theta_{0}=-2 \theta_{0}$, which leads to

$$
\left[\left(H_{0}-H\right)^{-1 / 2}\right] \quad \theta_{0}=105.2^{\circ}, P_{0}=.807,<H>=-0.212 .
$$

For the Boltzmann distribution we require

$$
\text { [Boltzmann] 2 } \alpha P_{0}=1.257, P_{0}=.809,<H>=-0.214 \text {. }
$$

Note, we have used $<H>=<\phi_{j}^{\prime 2}>/ 2-2 P_{0}<\cos \phi_{j}>=-P_{0}^{4} / 2$.

Remarkably, the value of $P_{0}$ is insensitive to the nature of the distribution. Furthermore, the simulation in Fig. 1.1 corresponds to an actual value $P_{0}=.8$, in excellent agreement with the prediction of the three distributions we have explored. Moreover, from Eq. (3.10), we find $\nu=P_{0}^{2} \approx 0.66$, in good agreement with the result $\nu=0.70$ given in Fig. 1.2 obtained from the simulation.

We can explore the electron distribution by comparing the results of simulations starting with a low radiation level with the three explicit distributions analyzed above. 
In Fig. 3.4 we show the three distributions as a function of $H$ defined in Eq. (3.11). In Fig. 3.5 we show the electron distributions obtained from the simulation for $\tau=10,20,30,40$. The background from the electrons which are not trapped is seen to be more or less independent of $H$, and the distributions of the trapped electrons seems to most resemble the Boltzmann distribution.

\section{Single Harmonic Model}

\subsection{First Order Treatment of Radiation Oscillations}

We now explore the oscillations about the equilibrium by assuming that only a single harmonic of relatively small amplitude is present. Thus we write

$$
\begin{gathered}
P(\tau)=P_{0}+P_{1} \cos \Omega \tau, \\
Q(\tau)=Q_{1} \sin \Omega \tau, \\
\beta_{j}(\tau)=\phi_{j}(\tau)+a \sin \Omega \tau,
\end{gathered}
$$

where we have assumed a coherent dipole oscillation of the electron phase space distribution. Expanding $<\sin \beta_{j}>$ to first order in $a$ and using Eq. (3.9), we find

$$
<\sin \beta_{j}(\tau)>=\bar{c} a \sin \Omega \tau
$$

where

$$
\bar{c} \equiv<\cos \phi_{j}>
$$

The first harmonic terms in Eqs. (3.4) and (3.5) now lead to

$$
\begin{gathered}
\Omega Q_{1}+(\nu-\delta) P_{1}=0 \\
(\nu-\delta) Q_{1}+\Omega P_{1}=-\bar{c} a
\end{gathered}
$$

from which we find

$$
Q_{1}=\frac{\bar{c}(\nu-\delta) a}{\Omega^{2}-(\nu-\delta)^{2}}, \quad P_{1}=-\frac{\bar{c} \Omega a}{\Omega^{2}-(\nu-\delta)^{2}} .
$$

We also average Eq. (3.3) over $j$ and obtain from the first harmonic

$$
\left(\Omega^{2}-2 P_{0} \bar{c}\right) a=2 \bar{c} Q_{1} .
$$


Combining Eqs. (4.8) and (4.9), we obtain

$$
\left(\Omega^{2}-2 P_{0} \bar{c}\right)\left(\Omega^{2}-(\nu-\delta)^{2}\right)=2 \bar{c}^{2}(\nu-\delta)=2 \bar{c}(\nu-\delta)^{2} P_{0},
$$

where the last form results from using Eq. (3.8). We then obtain a prediction for $\Omega$, the frequency of the oscillations about the equilibrium in the saturation regime

$$
\Omega^{2}=2 P_{0} \bar{c}+(\nu-\delta)^{2}=\left(P_{0}^{2}-\delta\right)\left(3 P_{0}^{2}-\delta\right),
$$

where we have used Eq. (3.18) for $\bar{c} \equiv<\cos \phi_{j}>$. For $\delta=0$, this corresponds to

$$
\Omega=\sqrt{3} P_{0}^{2} \cong 1.14[\text { Boltzmann]. }
$$

This prediction is somewhat smaller than the value $\Omega=1.25$ obtained from the simulation in Fig. 1.1. A more accurate determination of the coherent frequency $\Omega$ is given in Section 5 .

Finally, we can also use the first harmonic components of the two invariants. Not surprisingly, they each reproduce Eq. (4.11).

To summarize, we consider equilibrium solutions, and utilize the two invariants for an initial monoenergetic unbunched electron beam and a low initial level of radiation to determine the radiation parameters $\nu, P_{0}$ and the electron phase space averages $<\cos \phi_{j}>$ and $\left\langle\phi_{j}^{\prime 2}\right\rangle$. We then consider a first harmonic oscillation of the radiation and a coherent dipole oscillation of the electron distribution, from which we determine the oscillation frequency $\Omega$, as well as the relative oscillations amplitudes $P_{1}, Q_{1}, a$. The remaining question is to predict the magnitude of the amplitude of oscillation which, in fact, is not small.

\subsection{Transition Model}

An approximate model for the transition to the saturation regime is suggested by the plot of $P^{\prime}(\tau)$ vs. $P(\tau)$ in Fig. 4.1. It appears that the linear variation, corresponding to the exponential growth regime, is approximately tangent to the elliptical trajectories which correspond to the oscillations in the saturation regime. Quantitatively this requires that the logarithmic growth rate in the linear regime, given approximately by Eq. (2.7) for $\delta=0$, or its equivalent

$$
\frac{1}{P} \frac{d P}{d \tau} \cong \frac{\sqrt{3}}{2}
$$


is equal in the saturation regime to that of the osrillatory behavior of Eq. (4.1). This leads to the relation

$$
\frac{1}{\Omega} \frac{P^{\prime}}{P}=\frac{\sqrt{3}}{2 \Omega}=\frac{P_{1}}{\sqrt{P_{0}^{2}-P_{1}^{2}}}
$$

which suggests a value $P_{1} \cong 0.49$, about $20 \%$ larger than that seen in the simulation. Also we have from Eq. $(4.8),\left|Q_{1}\right|=(\nu / \Omega)\left|P_{1}\right| \cong 0.28$, about $30 \%$ larger than that seen in the simulation. However, the transition region is undoubtedly more complicated than the simple sudden change from one behavior to another, and the agreement is therefore reasonable.

Thus, we confirm the validity of our general picture of the way in which saturation sets in, although our small oscillation assumption is only approximately valid. In the next section, we explore the solutions of Eqs. (1.1)-(1.3) in the saturation region more rigorously.

\section{Small Oscillations About the Stationary Distri- butions}

\subsection{Action-Angle Variables}

We take the stationary state Hamiltonian to be

$$
H_{\circ}=\frac{\beta^{\prime^{2}}}{2}-2 P_{\circ} \cos \beta
$$

and introduce the radiation field variable $\tilde{P}(\tau)$, given by

$$
\tilde{P}(\tau)=P(\tau)-P_{o} .
$$

Equations (3.2) and (3.3) then become

$$
\beta^{\prime \prime}+2 P_{\circ} \sin \beta=-2[\tilde{P} \sin \beta+Q \cos \beta] .
$$

For $\delta=0$, Eqs. (3.4) and (3.5) lead to

$$
Q^{\prime}+\nu \tilde{P}=<\cos \beta>-\nu P_{0}
$$

and

$$
\tilde{P}-\nu Q=<\sin \beta>
$$


analogous to Eqs. (3.3)-(3.5). The Hamilunian corresponding to Eq. (5.3) is

$$
H=H_{0}+V
$$

where the perturbation $V$ is given by

$$
V(\tau)=2[\tilde{P}(\tau) \cos \beta-Q(\tau) \sin \beta] .
$$

The unperturbed stationary system corresponds to the action angle variables

$$
\begin{gathered}
I=\frac{1}{2 \pi} \oint d \beta \beta^{\prime}\left(\beta, H_{0}\right)=\frac{8 \sqrt{2 P_{0}}}{\pi}[E(m)-(1-m) K(m)], \\
\psi^{\prime} \equiv \omega(I) \equiv \frac{d H_{0}}{d I}=\frac{\pi \sqrt{2 P_{0}}}{2 K(m)}, H_{0}=4 P_{0} m-2 P_{0},
\end{gathered}
$$

where $m=\sin ^{2}(\theta / 2)$, with $\theta$ being the maximum pendulum angle corresponding to a given value of $I$. Here $P_{0}$ is considered as a constant, and the stationary phase space distribution in the $I, \psi$ space is taken to be

$$
f_{\text {stationary }}\left(\beta, \beta^{\prime}\right)=f_{0}(I) \text {. }
$$

The solutions of the pendulum system can be written in terms of the Jacobi elliptic functions as

$$
\begin{gathered}
\beta=2 \sin ^{-1}\left\{\sqrt{m} \operatorname{sn}\left[\left(\frac{2}{\pi} K(m) \psi\right]\right\},\right. \\
\beta^{\prime}=\sqrt{8 P_{\circ} m} \operatorname{cn}\left[\left(\frac{2}{\pi}\right) K(m) \psi\right] .
\end{gathered}
$$

Using the expansion of the periodic functions $s n, c n$ into Fourier series ${ }^{9}$ :

$$
\begin{aligned}
& \operatorname{sn}\left(\frac{2}{\pi} K(m) \psi\right)=\frac{2 \pi}{\sqrt{m} K} \sum_{n=0}^{\infty} \frac{q^{n+\frac{1}{2}}}{1-q^{2 n+1}} \sin (2 n+1) \psi, \\
& c n\left(\frac{2}{\pi} K(m) \psi\right)=\frac{2 \pi}{\sqrt{m} K} \sum_{n=0}^{\infty} \frac{q^{n+\frac{1}{2}}}{1+q^{2 n+1}} \cos (2 n+1) \psi,
\end{aligned}
$$

where

$$
q=\exp \left(\frac{-\pi K(1-m)}{K(m)}\right)=\frac{m}{16}\left[1+\frac{m}{2}+\frac{21 m^{2}}{64}+\cdots\right]
$$

we can write for the harmonic decomposition of the pendulum motion

$$
\begin{gathered}
\cos \beta=\sum_{n=0}^{\infty} A_{n}(I) \cos 2 n \psi, \\
\sin \beta=\sum_{n=0}^{\infty} B_{n}(I) \sin (2 n+1) \psi,
\end{gathered}
$$

where $A_{n}(I)$ and $B_{n}(I)$ are related to the coefficients in Eqs. (5.13) and (5.14). 


\subsection{Perturbation Treatment and Dispersion Relation}

The behavior of the perturbed system is governed by the Vlasov equation

$$
\frac{\partial f}{\partial \tau}+\frac{\partial H}{\partial I} \frac{\partial f}{\partial \psi}-\frac{\partial H}{\partial \psi} \frac{\partial f}{\partial I}=0 \text {. }
$$

In Appendix A we explore oscillations about the equilibrium solution by treating $f-f_{0}, \tilde{P}(\tau)$ and $Q(\tau)$ as small quantities. This linearized system has oscillat modes with frequency $\Omega$ given by the dispersion relation

$$
\left[\nu-\sum_{n=1}^{\infty} S_{n}(\Omega)\right]\left[\nu-\sum_{n=0}^{\infty} T_{n}(\Omega)\right]=\Omega^{2}
$$

where

$$
\begin{gathered}
S_{n}(\Omega)=2 \pi \int d I f_{0}(I) \frac{d}{d I}\left[\frac{(2 n)^{2} \omega A_{n}^{2}}{(2 n)^{2} \omega^{2}-\Omega^{2}}\right], \\
T_{n}(\Omega)=2 \pi \int d I f_{0}(I) \frac{d}{d I}\left[\frac{(2 n+1)^{2} \omega B_{n}^{2}}{(2 n+1)^{2} \omega^{2}-\Omega^{2}}\right] .
\end{gathered}
$$

Since $A_{n}$ and $B_{n}$, defined in Eqs. (5.16) and (5.17), are real, stability requires that all solutions for $\Omega$ in Eq. (5.19) be real.

For the normalized delta function (KV) distribution

$$
f_{0}(I)=\frac{1}{2 \pi} \delta\left(I-I_{0}\right)
$$

corresponding to Eq. (3.12), we have

$$
\begin{gathered}
S_{n}(\Omega)=\omega \frac{d}{d H_{0}}\left[\frac{(2 n)^{2} \omega A_{n}^{2}}{(2 n)^{2} \omega^{2}-\Omega^{2}}\right] \\
T_{n}(\Omega)=\omega \frac{d}{d H_{0}}\left[\frac{(2 n+1)^{2} \omega B_{n}^{2}}{(2 n+1)^{2} \omega^{2}-\Omega^{2}}\right]
\end{gathered}
$$

where $I=I_{0}$ is given in Eq. (5.8) and where the relation between $I_{0}, H_{0}$ and $m$ is given in Eq. (5.9), with $P_{0}$ taken as a constant. Thus $d / d H_{0}=\left(1 / 4 P_{0}\right) d / d m$, and the derivatives in Eqs. (5.22) and (5.23) act on $\omega, A_{n}(I), B_{n}(I)$, where $\omega$ and $I$ are expressed in terms of $m$ in Eqs. (5.8), (5.9).

The exact solutions to Eq. (5.19), with $S_{n}(\Omega), T_{n}(\Omega)$ given by Eqs. (5.23), (5.24) can only be obtained numerically. Moreover, the discussion in Section 3 and the corresponding simulations show that the electron bunch will be relatively large, corresponding to a relatively large value of $\theta_{0}$, the maximum pendulum angle. Nevertheless we can obtain a guide to the location and stability of the oscillation modes, 
as well as a useful starting value for the numerical search for these modes, by exploring approximate analytic solutions corresponding to the $\mathrm{KV}$ distribution for small $m=\sin ^{2}\left(\theta_{0} / 2\right)$.

We start by expanding $A_{n}$ and $B_{n}$ in powers of $m$. Specifically we find

$$
\begin{gathered}
A_{n} \equiv G_{2 n} \cong \frac{16 n m^{n}}{4^{2 n}}, n \geq 1, \\
B_{n} \equiv G_{2 n+1} \cong \frac{8(2 n+1)}{4^{2 n+1}} m^{n+\frac{1}{2}}, n \geq 0 .
\end{gathered}
$$

The dispersion equation can then be written more compactly as

$$
\left(\nu-\sum_{\substack{p=2 \\ \text { even }}} H_{p}\right)\left(\nu-\sum_{\substack{p=1 \\ \text { odd }}} H_{p}\right)=\Omega^{2}
$$

with

$$
H_{p}=\frac{\omega}{4 P_{0}} \frac{d}{d m}\left[\frac{p^{2} \omega G_{p}^{2}}{p^{2} \omega^{2}-\Omega^{2}}\right], p \geq 1
$$

where

$$
\omega(m)=\frac{\pi \sqrt{2 P_{0}}}{2 K(m)}
$$

Since $G_{p}^{2} \sim m^{p}$, the dominant term for small $m$ is the one for $p=1$. In this approximation, Eq. (5.27) becomes

$$
\nu\left(\nu-H_{1}\right) \cong \Omega^{2}
$$

with

$$
H_{1} \cong \frac{\omega^{2}}{4 P_{0}\left(\omega^{2}-\Omega^{2}\right)} \frac{d G_{1}^{2}}{d m} \cong \frac{\omega^{2}}{P_{0}\left(\omega^{2}-\Omega^{2}\right)}
$$

and

$$
\omega(0)=\sqrt{2 P_{o}}
$$

According to Eq. (5.4), $\nu P_{0} \cong 1$ for an equilibrium distribution with small $\theta_{0}$. As a result Eqs. (5.30)-(5.32) lead to

$$
\Omega^{2}\left[\Omega^{2}-\left(2 P_{o}+\nu^{2}\right)\right]=0
$$

predicting roots at

$$
\Omega_{d}^{2} \cong 2 P_{0}+\nu^{2}, \Omega_{o}^{2} \cong 0
$$

The root $\Omega_{d}$ corresponds to a dipole-like oscillation of both the phase space density and the radiation. In fact, this is the small angle limit of the dipole root in 14.11 ) 
obtained without a self consistent calculation. A more accurate calculation of the root $\Omega_{0}$ requires additional terms in the expansion near $m=0$. Both roots turn out to be real for small $\theta_{0}$ implying stability of the equilibrium distribution for these modes.

The singular behavior of $H_{p}$ implies the existence of additional roots of Eq. (5.27) near $\Omega_{p}^{2}=p^{2} \omega^{2} \cong 2 p^{2} P_{o}$. Inclusion of appropriate terms for small $\theta_{o}$ leads to the conclusion that the root $\Omega_{1}$ is real but that the roots $\Omega_{p}, p \geq 2$, each have a small imaginary part proportional to $\theta_{0}^{p}$. These roots appear to be associated with a phase space distribution where there are $p$ wiggles along the boundary of the distribution. The growth rate for these modes is expected to be slow for small $\theta_{0}$. These predictions appear to be consistent with the exact numerical solutions of Eq. (5.27) as well as with several numerical simulations illustrating the modes. This numerical work is described in the next section.

\section{Numerical Results}

In Figs. 6.1, 6.2, we show the result of a simulation to check the equilibrium solution for a small pendulum angle $\theta_{0}=3^{\circ}$. Figure 6.1 shows the corresponding stationary phase space distribution and Fig. 6.2 shows the equilibrium value of $P(\tau)=P_{0}=$ 1.00 .

In Fig. 6.3 we explore the oscillation of $|A(\tau)|$ which occurs when we start with the phase space distribution in Fig. 6.1, but with $P(0) / P_{0}=0.99$. The dipole oscillation with frequency $\left(2 P_{o}+\nu^{2}\right)^{1 / 2}=1.73$ shows clearly, and appears to be stable. A similar result is shown in Fig. 6.4 for $\theta_{0}=30^{\circ}$ with $P(0) / P_{0}=0.99$. But the simulations for $\theta_{0}=55^{\circ}$, and $80^{\circ}$, shown in Fig. 6.5 and 6.6 show an unstable dipole oscillation.

We then obtained the numerical solution of the dispersion equation, Eq. (5.19), and this is displayed in Fig. 6.7, where we have only included the dipole term $T_{0}$. Clearly an instability is predicted for $\theta_{0}>50^{\circ}$, consistent with our observations in Figs. 6.3-6.6. We then included several additional terms in Eq. (5.19) and the results are shown in Fig. 6.8. The additional modes, near $\Omega_{p}^{2} \cong 2 p^{2} P_{o}$ for small $\theta_{o}$, show up clearly for $p=2$ and $p=3$, but it appears that these modes are unstable at all values of $\theta_{0}$. But our starting phase space distribution does not contain the "border ripples" corresponding to these modes and therefore they are not seen in the simulations. Note also the stable mode with small frequency in Fig. 6.7, as predicted in Eq. (5.34). 
It is interesting to note that the unstable modes in Figs. 6.5 and 6.6 saturate. This is in fact reassuring since the dipole mode obtained in Section 4 does not exhibit unstable behavior. Thus it appears that the instabilities associated with the roots of Eq. (5.19) are not in conflict with the observation of saturation of the FEL radiation with a significant dipole oscillation. From Fig. 6.6, we see that the $\operatorname{Re} \Omega=1.25$, in agreement with the start-up simulation of Fig. 1.1. The dispersion relition results presented in Fig. 6.7 predict that for $\theta_{0}=81^{\circ}, \operatorname{Re} \Omega=1.22$, in good agreement with the computer simulation.

Finally, we include the prediction of Eqs. (3.8), (3.10), (3.13) and (4.11) for $\delta=0$ to obtain

$$
\begin{aligned}
\Omega^{2}=3 P_{0}^{4}, & <\cos \phi_{j}>=P_{0}^{3}=\frac{2 E(m)}{K(m)}-1 \\
\Omega^{2} & =3\left[\frac{2 E(m)}{K(m)}-1\right]^{4 / 3} .
\end{aligned}
$$

The curve corresponding to Eq. (6.2) is included as a dashed line in Fig. 6.7. The result is quite close to the exact solution for smal' $\theta_{0}$, and is a suprisingly good approximation for all $\theta_{0}$.

\section{Effect of Detuning, Energy Spread}

We return to the treatment in Section 4 to explore the effect of an initial detuning. and energy spread of the electron beam. The main change is to include the constant $C_{2}=\epsilon^{2} / 2$ in Eqs. (1.5) and (3.7), where

$$
\epsilon^{2}=<p_{j}^{2}>\left.\right|_{\tau=0}
$$

is the initial mean square (scaled) energy spread. Thus, for the Boltzmann distribution we find from Eqs. (3.7), (3.8), (3.10) and (3.17)

$$
\begin{gathered}
<\phi_{j}^{\prime 2}>=\frac{1}{\alpha}=3 P_{0}^{4}-2 \delta P_{0}^{2}+\epsilon^{2}, \\
<\cos \phi_{j}>=\frac{I_{1}\left(2 \alpha P_{0}\right)}{I_{0}\left(2 \alpha P_{0}\right)}=P_{0}\left(P_{0}^{2}-\delta\right)
\end{gathered}
$$

The solution of Eq. (7.2) and (7.3) for $P_{0}$ as a function of $\delta$, for $\epsilon^{2}=0$, is shown as the solid curve in Fig. 7.1. In addition we show results for simulations with 
$\epsilon^{2}=0$, which agree reasonably well with the predictions. At this point we should point out that Eqs. (7.2) and (7.3) have been derived under the assumptions that all electrons have been captured by the bunch. In fact, only about $80 \%$ of the electrons are captured for $\epsilon^{2}=0$. If we assume that the $20 \%$ which have not been captured are uniformly distributed in $\phi_{j}$ and have values of $p_{j}$ which remain small, we estimate that $P_{0}$ would be reduced from the value predicted by Eqs. (7.2) and (7.3) by about $6 \%$ for $\delta=0$, which may be the reason the simulations for $\epsilon^{2}=0$ are mostly below the curve.

The situation is somewhat more ambiguous for $\epsilon^{2}=0.5$, where only about $70 \%$ of the electrons are captured, and for $\epsilon^{2}=1.0$ where only about $50 \%$ of the electrons are captured. We see clearly from the simulations that $P_{0}$ decreases as $\epsilon^{2}$ increases. Any analytic treatment would require some way of predicting the fraction of the electrons which are captured as a function of $\delta$ and $\epsilon^{2}$, as well as the way in which Eqs. (7.2) and (7.3) should be modified to take into account the untrapped electrons. This is a subject for future study.

\section{Electron Beam with Finite Radial Extent}

We shall now extend the single harmonic model introduced in Sections 3 and 4 for the one-dimensional FEL dynamics to the two-dimensional case of an electron beam with finite radial extent. We ignore betatron oscillations, assuming the electron beam. has no angular spread, but include the diffraction of the radiation and the radiation focusing properties of the electron beam bunched by the FEL interaction.

\subsection{Equations of Motion and Invariants}

We consider a fixed electron beam density profile, $u(r)$. The equations for the electron motion are

$$
\begin{gathered}
\sigma_{j}^{\prime}=p_{j}, \\
p_{j}^{\prime}=-A e^{i \sigma_{j}}-A^{*} e^{-i \sigma_{j}},
\end{gathered}
$$

where the scaled amplitude of the radiation $A(r, \tau)$ depends on both the scaled longitudinal position $\tau=2 \rho k_{w} z$ and the scaled transverse coordinates $\vec{r}=\sqrt{4 \rho k_{w} k_{s}} \vec{r}_{d}$, 
where $k_{s}$ is the resonant radiation wave number and $\vec{r}_{d}$ is the unscaled transverse coordinate vector. The wave equation in the case of zero detuning is

$$
A^{\prime}-i \nabla^{2} A=u(r)<e^{-i \sigma_{j}}>
$$

where $\nabla^{2}=\partial^{2} / \partial r_{1}^{2}+\partial^{2} / \partial r_{2}^{2}$ is the two-dimensional Laplacian in the scaled transverse coordinates $\vec{r}=\left(r_{1}, r_{2}\right)$, and $r=\sqrt{r_{1}^{2}+r_{2}^{2}}$ is the magnitude of the vector $\vec{r}$.

Eqs. (8.1)-(8.3) have two invariants of the motion. We can use Eqs. (8.2) and (8.3) to obtain

$$
u(r)<p_{j}^{\prime}>=-\frac{\partial}{\partial \tau}\left(A A^{*}\right)-i \nabla \cdot\left(A \nabla A^{*}-A^{*} \nabla A\right) .
$$

Integrating over $d^{2} r$ then leads to the vanishing of the divergence term, and the first invariant

$$
\int_{0}^{\infty} r d r\left[<p_{j}>u(r)+|A|^{2}\right]=C_{1} .
$$

A similar analysis leads to the second invariant

$$
\int_{0}^{\infty} r d r\left[<p_{j}^{2}>u(r)-2 i\left(A A^{\prime *}-A^{*} A^{\prime}\right)-2|\nabla A|^{2}\right]=C_{2} .
$$

Comparing these results with the one-dimensional invariants of Eqs. (1.4) and (1.5), we see there is a new term in the second invariant, $|\nabla A|^{2}$, and the electron averages are weighted by the transverse electron beam density. Each invariant is expressed as an integral over the electron beam and radiation radial densities.

As in the one-dimensional treatment of saturation, we extract a phase factor and write

$$
A(r, \tau)=[P(r, \tau)+i Q(r, \tau)] e^{i \nu\left(r-\tau_{0}\right)},
$$

and introduce the electron phase $\beta_{j}$ given by

$$
\beta_{j}=\sigma_{j}+\nu\left(\tau-\tau_{0}\right)+\pi / 2
$$

We asume $\nu$ is independent of $r$; hence using Eq. (8.3) we can write ${ }^{8}$

$$
\begin{gathered}
Q^{\prime}+\nu P-\nabla^{2} P=u(r)<\cos \beta_{j}>, \\
-P^{\prime}+\nu Q-\nabla^{2} Q=-u(r)<\sin \beta_{j}>.
\end{gathered}
$$

The equation for the electron phase becomes

$$
\beta_{j}^{\prime \prime}=-2 P \sin \beta_{j}-2 Q \cos \beta_{j},
$$


and averaging over the electron longitudinal phase space distribution at each radius, we obtain

$$
<\beta_{j}^{\prime \prime}>=-2 P<\sin \beta_{j}>-2 Q<\cos \beta_{j}>\text {. }
$$

The first and second invariants are written as

$$
\int_{0}^{\infty} r d r\left[<\beta_{j}^{\prime}>u+P^{2}+Q^{2}-\nu u\right]=C_{1}
$$

and

$$
\int_{0}^{\infty} r d r\left[<\beta_{j}^{\prime 2}>u-\nu^{2} u-2 \nu\left(P^{2}+Q^{2}\right)-2(\nabla P)^{2}-2(\nabla Q)^{2}-4\left(P Q^{\prime}-Q P^{\prime}\right)\right]=C_{2} .
$$

The constants $C_{1}$ and $C_{2}$ are taken as zero corresponding to an initial electron distribution with the correct energy and no energy spread and a very low initial radiation amplitude.

\subsection{Equilibrium Solutions}

We now consider the description of the equilibrium state. Let $\phi_{j}$ denote an equilibrium electron phase distribution, and take $P=P_{0}(r)$ and $Q=0$. From Eqs. (8.9) and (8.10) we find

$$
\begin{gathered}
\nu P_{o}(r)-\nabla^{2} P_{0}=u(r)<\cos \phi_{j}> \\
<\sin \phi_{j}>=0 .
\end{gathered}
$$

Utilizing $\left\langle\phi_{j}^{\prime}\right\rangle=0$ and the first and second invariants of Eqs. (8.13) and (8.14) with $C_{1}=C_{2}=0$, we obtain

$$
\begin{gathered}
\int_{0}^{\infty} r d r P_{0}^{2}=\nu \int_{0}^{\infty} r d r u \\
\int_{0}^{\infty} r d r<\phi_{j}^{\prime 2}>u=\int_{0}^{\infty} r d r\left[\nu^{2} u+2 \nu P_{0}^{2}+2\left(\nabla P_{0}\right)^{2}\right]
\end{gathered}
$$

It follows from Eq. (8.11) that for any given $r$, any function of

$$
H\left(\phi_{j}, \phi_{j}^{\prime} ; r\right)=\phi_{j}^{2} / 2-2 P_{0}(r) \cos \phi_{j}
$$

will be a stationary distribution. For example, one could consider the KV distribution

$$
f_{K V}\left(\phi_{j}, \phi_{j}^{\prime} ; r\right)=N(r) \delta\left(2 P_{0}(r) \cos \phi_{j}-\phi_{j}^{\prime 2} / 2-2 P_{0}(r) \cos \theta_{0}(r)\right)
$$


In this case

$$
\begin{gathered}
\bar{c}(r) \equiv<\cos \phi_{j}>=\frac{2 E(m)}{K(m)}-1, m=\sin ^{2} \frac{\theta_{0}(r)}{2} \\
<\phi_{j}^{2}>=4 P_{0}(r)\left(\bar{c}(r)-c_{0}(r)\right), \quad c_{0}(r)=\cos \theta_{0}(r)=1-2 m .
\end{gathered}
$$

In the discussion which follows, we shall explore the consequences of the simplifying assumption that $\theta_{0}$ is independent of $r$, which of course also implies $m, c$ and $c_{0}$ are $r$-independent. In this case Eq. (8.15) becomes

$$
\nu_{\nu_{0}}(r)-\nabla^{2} P_{0}=u(r) \bar{c}
$$

and the invariants can be expressed in the form

$$
\nu=\frac{\int_{0}^{\infty} r d r P_{0}^{2}}{\int_{0}^{\infty} r d r u}
$$

and

$$
\nu^{2}=\frac{2\left(\bar{c}-2 c_{0}\right) \int_{0}^{\infty} r d r u P_{0}}{\int_{0}^{\infty} r d r u}
$$

where we have used Eq. (8.23) in Eq. (8.18) to derive Eq. (8.25).

We now consider the electron beam density to be given by

$$
u(r)=\left\{\begin{array}{ll}
1, & r<R \\
0, & r>R
\end{array}\right\}
$$

In this case the solution of Eq. (8.23) is

$$
P_{0}(r)=\left\{\begin{array}{cc}
(\bar{c} / \nu)\left[1-x K_{1}(x) I_{0}(\sqrt{\nu} r)\right], & r \leq R \\
(\bar{c} / \nu) x I_{1}(x) K_{0}(\sqrt{\nu} r), & r \geq R
\end{array}\right\}
$$

where $x=\sqrt{\nu} R$. The two invariants of Eqs. (8.24) and (8.25) can be expressed as

$$
\begin{gathered}
\frac{\nu^{3}}{\bar{c}^{2}}=2 x K_{1}(x) I_{2}(x), \\
\frac{\nu^{3}}{\bar{c}\left(\bar{c}-2 c_{0}\right)}=2 x K_{1}(x) I_{2}(x)+2 x I_{1}(x) K_{0}(x) .
\end{gathered}
$$

From Eqs. (8.28), (8.29), we obtain

$$
\frac{\bar{c}}{2 c_{0}}=1+\frac{I_{2}(x) K_{1}(x)}{I_{1}(x) K_{0}(x)}
$$

The one-dimensional results of Section 3 are recovered in the limit $x=\sqrt{\nu} R \rightarrow \infty$. From Eq. (8.27) it is seen that $P_{0}(r) \cong \bar{c} / \nu \equiv P_{0}$ (independent of $r$ ) inside the electron 
beam, and $P_{0}(r)=0$ outside the electron beam, except in a region $\Delta r \sim \nu^{-1 / 2}$ near the edge $r=R$. In the $x \rightarrow \infty$ limit, Eq. (8.28) becomes $\nu^{3}=\bar{c}^{2}$. Hence, using $\bar{c}=\nu P_{0}$ we find $\nu=P_{0}^{2}$ and $\bar{c}=P_{0}^{3}$, the one-dimensional results. Also, when $x \rightarrow \infty$, Eq. (8.30) implies $\bar{c}=4 c_{0}$ which is the one-dimensional result of Eq. (3.21), so one obtains the one-dimensional values $m=0.433, \theta_{0}=82.3^{\circ}$ and $P_{0}=0.813$ given in Eq. (3.22) for the KV distribution.

\subsection{Single Harmonic Model}

As in the one-dimensional case discussed in Section 4, we assume that the radiation amplitude oscillates with a single harmonic aisst the equilibrium value, but in this case the equilibrium value as well as the oscillation amplitude depend on radius. We assume, however, that the oscillation frequency is independent of radius. Thus we write

$$
\begin{gathered}
P(r, \tau)=P_{0}(r)+\xi(r) e^{i \Omega \tau}+\xi^{*}(r) e^{-i \Omega^{*} \tau}, \\
Q(r, \tau)=\eta(r) e^{i \Omega \tau}+\eta^{*}(r) e^{-i \Omega^{*} \tau}
\end{gathered}
$$

where $\Omega$ may have a positive imaginary part describing the damping of the oscillations resulting from the radiation of energy out of the region occupied by the electron beam. The spirit of Eqs. (8.31) and (8.32) is that $\xi(r)$ and $\eta(r)$ are first-order, and we neglect second-order terms. Moreover, we ignore second and higher harmonics.

We make a similar assumption about the electron phase $\beta_{j}$, writing

$$
\beta_{j}=\phi_{j}+a(r) e^{i \Omega \tau}+a^{*}(r) e^{-i \Omega^{*} \tau},
$$

where $\phi_{j}$ is the equilibrium phase discussed in Section 8.2 , and $a(r)$ is the first-order amplitude of the coherent dipole oscillation mode of the electron beam in longitudinal phase space. We also employ the approximations

$$
\begin{gathered}
<\cos \beta_{j}>=<\cos \phi_{j}>=\bar{c} \\
<\sin \beta_{j}>=\bar{c}\left[a(r) e^{i \Omega \tau}+a^{*}(r) e^{-i \Omega^{*} \tau}\right],
\end{gathered}
$$

Using expansions of Eqs. (8.31)-(8.35) in the wave Eqs. (8.9) and (8.10), we find

$$
\left(\nabla^{2}-\nu\right) \xi(r)=i \Omega \eta(r)
$$




$$
\left(\nabla^{2}-\nu\right) \eta(r)=-i \Omega \xi(r)+\bar{c} u(r) a(r) .
$$

Similariy, utilizing the expansions of Eqs. (8.31)-(8.35) in the pendulum Eq. (8.12), we obtain

$$
\left[\Omega^{2}-2 \bar{c} P_{0}(r)\right] a(r)=-2 \bar{c} \eta(r) .
$$

Now eliminating $a(r)$ in Eq. (8.37) by using Eq. (8.38), we derive

$$
\left[\nabla^{2}-\nu-2 \lambda(r)\right] \eta(r)=-i \Omega \xi(r)
$$

where

$$
\lambda(r)=\frac{\bar{c}^{2} u(r)}{\Omega^{2}-2 \bar{c} P_{0}(r)} .
$$

The coherent oscillation frequency $\Omega$ and the oscillation amplitudes $\xi(r)$ and $\eta(r)$ are determined from solving the eigenvalue problem specified by Eqs. (8.36) and (8.39).

\subsection{Large Electron Beam Radius}

In order to proceed further, we must now obtain the eigenvalue $\Omega$ from the solution of Eqs. (8.36) and (8.39). Since $\lambda(r)$ in Eq. (8.40) depends on $r$, even under the assumption that $c$ is independent of $r$, solution of the eigenvalue problem is difficult. However, results can be obtained in a straightforward manner in the limit $R \rightarrow \infty$. We shall examine this limit to see if physically sensible results are obtained.

When $x=\sqrt{\nu} R$ in Eq. (8.27) is much larger than unity, we find that $P_{0}(r)=$ $\bar{c} / \nu=P_{0}$ (independent of $r$ ) inside the electron beam and $P_{0}(r)=0$ outside the electron beam, except for a region $\Delta r \sim \nu^{-1 / 2}$ near the edge $r=R$. Hence, for $x \gg 1$ we can make the approximation

$$
\lambda(r)=\left\{\begin{array}{ll}
\lambda, & r<R \\
0, & r>R
\end{array}\right\},
$$

where

$$
\lambda=\frac{\bar{c}^{2}}{\Omega^{2}-2 \bar{c} P_{0}} .
$$

Furthermore, for large $x$, Eqs. (8.28) and (8.29) lead to the one-dimensional results $\nu=P_{0}^{2}, \bar{c}=P_{0}^{3}$ with

$$
[\mathrm{KV}] \quad m=0.433, \theta_{0}=82.3^{\circ}, P_{0}=0.813
$$


as given in Eq. (3.22).

We now must solve Eqs. (8.36) and (8.39) for constant $\lambda$ in order to obtain an equation for the frequency $\Omega$. Outside the electron beam we have from Eqs. (8.36) and (8.39) with $\lambda(r)=0$,

$$
\nabla^{2}(\xi \pm i \eta)= \pm(\Omega \pm \nu)(\xi \pm i \eta), \quad r>R
$$

so that

$$
\begin{array}{ll}
2 \xi=A K_{0}(p r / R)+B H_{0}^{(2)}(q r / R), & r>R, \\
2 \eta=A K_{0}(p r / R)-B H_{0}^{(2)}(q r / R), & r>R,
\end{array}
$$

where

$$
p=\sqrt{\Omega+\nu} R, q=\sqrt{\Omega-\nu} R,
$$

and where $H_{0}^{(2)}$ has been chosen to correspond to an outgoing wave. Note that there is both a guided and propagating component in Eqs. (8.45) and (8.46).

Inside the electron beam, we also have two Bessel function solutions, which depend in this case on $\lambda$ given in Eq. (8.42). We write

$$
\begin{gathered}
\xi=C I_{0}(\alpha r / R)+D J_{0}(\beta r / R) \\
i \Omega \eta=\left(\alpha^{2} / R^{2}-\nu\right) C I_{0}(\alpha r / R)-\left(\beta^{2} / R^{2}+\nu\right) D J_{0}(\beta r / R)
\end{gathered}
$$

where

$$
\begin{aligned}
& \alpha^{2} / R^{2}=\sqrt{\Omega^{2}+\lambda^{2}}+\nu+\lambda, \\
& \beta^{2} / R^{2}=\sqrt{\Omega^{2}+\lambda^{2}}-\nu-\lambda .
\end{aligned}
$$

Continuity of $\xi, \eta, d \xi / d r, d \eta / d r$ at $r=R$ leads after considerable algebra to

$$
\frac{\left(\alpha^{2}+q^{2}\right)\left(\beta^{2}+p^{2}\right)}{\left(\alpha^{2}-p^{2}\right)\left(q^{2}-\beta^{2}\right)}=\frac{(\hat{J}-\hat{K})(\hat{I}-\hat{H})}{(\hat{I}-\hat{K})(\hat{H}-\hat{J})}
$$

where

$$
\begin{gathered}
\hat{K}=\frac{p K_{0}^{\prime}(p)}{K_{0}(p)}, \hat{H}=\frac{q H_{0}^{(2)^{\prime}}(q)}{H_{0}^{(2)}(q)} \\
\hat{I}=\frac{\alpha I_{0}^{\prime}(\alpha)}{I_{0}(\alpha)}, \hat{J}=\frac{\beta J_{0}^{\prime}(\beta)}{J_{0}(\beta)}
\end{gathered}
$$


In the limit $R \rightarrow \infty$, it turns out that $p, q, \alpha$ each approach $\infty$, but $\beta$ remains finite. To confirm this, we use the limiting values

$$
\hat{K} \rightarrow-p, \hat{H} \rightarrow-i q, \hat{I} \rightarrow \alpha, \text { as } R \rightarrow \infty
$$

and neglect $\beta$ compared with $p, q, \alpha$ in all terms except $\hat{J}$. In this way we find

$$
\frac{\hat{J}+p}{\hat{J}+i q}=\frac{-(\alpha-i q) p^{2}}{(\alpha-p) q^{2}}
$$

or

$$
\frac{1}{\hat{J}}=\frac{J_{0}(\beta)}{\beta J_{0}^{\prime}(\beta)}=\frac{1}{\alpha}-\frac{1}{p}+\frac{i}{q} .
$$

Since $\alpha^{-1}, p^{-1}, q^{-1}$ approach zero proportionally to $R^{-1}$, we see that $\beta \rightarrow s_{m}$, the zeros of $J_{0}(s)$. In fact, setting $\beta=s_{1}+\delta$, where $s_{1}=2.405$, we find

$$
\beta \cong s_{1}\left(1+\frac{1}{\alpha}-\frac{1}{p}+\frac{i}{q}\right) \text {. }
$$

Using the one-dimensional values $\nu=P_{0}^{2}, \bar{c}=P_{0}^{3}, \Omega=\sqrt{3} P_{0}^{2}$, we find $\lambda=P_{0}^{2}$ [Eq. (8.42)], $\alpha^{2}=4 P_{0}^{2} R^{2}$ [Eq. (8.50)], $p^{2}=(\sqrt{3}+1) P_{0}^{2} R^{2}$ [Eq. (8.47)], and $q^{2}=$ $(\sqrt{3}-1) P_{0}^{2} R^{2}$ [Eq. (8.47)]. The terms in $\alpha^{-1}$ and $p^{-1}$ are small compared to unity and can therefore be ignored in Eq. (8.58). However, the term in $q^{-1}$ introduces damping arising from electromagnetic energy propagating out of the region occupied by the electron beam. The net frequency shift due to the finite, but large, electron beam radius is obtained from Eqs. (8.50) and (8.51), writing

$$
\beta^{2} \alpha^{2}=R^{4}\left(\Omega^{2}-\nu^{2}-\frac{2 \nu \bar{c}^{2}}{\Omega^{2}-2 \bar{c} P_{0}}\right) .
$$

Using Eq. (8.58) together with $\bar{c}=P_{0}^{3}, \nu=P_{0}^{2}$, Eq. (8.59) can be rewritten as

$$
\frac{\Omega^{2}}{\Omega^{2}-2 P_{0}^{4}}\left(\Omega^{2}-3 P_{0}^{4}\right) \cong \frac{s_{1}^{2} \alpha^{2}}{R^{4}}\left(1+\frac{2 i}{q}\right) \text {. }
$$

Finally we obtain

$$
\Omega \cong \sqrt{3} P_{0}^{2}+\frac{2 s_{1}^{2}}{3 \sqrt{3} R^{2}}\left(1+\frac{2 i}{P_{0} R(\sqrt{3}-1)^{1 / 2}}\right),
$$

corresponding to a frequency shift proportional to $R^{-2}$ and a damping rate proportional to $R^{-3}$. 


\subsection{Small Electron Beam Radius}

Let us now consider the limit $x=\sqrt{\nu} R \rightarrow 0$. The small argument approximations to the Bessel functions are:

$$
\begin{gathered}
I_{1}(x) \approx \frac{x}{2}, K_{1}(x) \approx \frac{1}{x}-\frac{x L}{2}, \\
I_{2}(x) \approx \frac{x^{2}}{8}, K_{0}(x) \approx 4\left[\ln (2 / x)-\gamma_{E}\right],
\end{gathered}
$$

where $\gamma_{E}=0.577$ is the Euler constant and

$$
L=\ln (2 / x)+\frac{1}{2}-\gamma_{E}
$$

When $x=\sqrt{\nu} R$ is much smaller than unity, we seen from Eq. (8.27) that $P_{0}$ is approximately constant within the electron beam. Specifically, it has the limiting form

$$
P_{0} \approx \frac{\bar{c} R^{2} L}{2}
$$

Using Eq. (8.28), we derive

$$
\nu \approx \frac{\bar{c} R}{2},
$$

and from Eq. (8.30), neglecting $L^{-1}$ compared to unity,

$$
\bar{c} \approx 2 c_{0} \text {. }
$$

Now employing Eqs. (8.21) and (8.22) one obtains

$$
\theta_{0}=71^{\circ}, c_{0}=0.32, \bar{c}=0.64 \text {. }
$$

We recall ${ }^{1}$ that the scaled electric field $A$ is related to the actual electric field $E$ by

$$
|E|^{2}=4 \pi \rho n_{0} W|A|^{2}
$$

where $n_{0}$ is the peak density of the electron beam and $W=\gamma m c^{2}$ is the average electron energy, with $c$ being the velocity of light. The power in the guided radiation is

$$
P_{\text {rad }}=\int_{0}^{\infty} 2 \pi r_{d} d r_{d} \frac{c}{4 \pi}|E|^{2},
$$

where the dimensioned radial coordinate $r_{d}$ is related to the scaled radial coordinate $r$ via

$$
r=\sqrt{4 \rho k_{w} k_{s}} r_{d} .
$$


Using Eqs. (8.66) and (8.68) in Eq. (8.67) leads to

$$
P_{\text {rad }}=\frac{c n_{0} W}{4 k_{w} k_{s}} \int_{0}^{\infty} 2 \pi r d r P_{0}^{2}
$$

From Eq. (8.24), we observe

$$
2 \pi \int_{0}^{\infty} r d r P_{0}^{2}=2 \pi \nu \int_{0}^{\infty} r d r u \approx \frac{\bar{c} R}{2}\left(\pi R^{2}\right)
$$

where Eq. (8.63) has been used. It now follows that

$$
P_{\text {rad }}=\nu \rho P_{e} \approx \frac{\bar{c}}{4} D P_{e},
$$

where $P_{e}$ is the electron beam power,

$$
P_{e}=I_{0} W / e
$$

$I_{0}$ is the electron current,

$$
I_{0}=e n_{0} c \pi R_{d}^{2}
$$

$R_{d}$ is the dimensioned electron beam radius related to the scaled radius by 10,11

$$
R^{2}=4 \rho k_{s} k_{-\nu} R_{d}^{2}
$$

and (niks units)

$$
D=2 \rho R=\left[\frac{2 e Z_{0}}{\pi m c^{2}} \frac{K^{2}}{1+K^{2}} \frac{I_{0}}{\gamma}\right]^{1 / 2},
$$

is the scaled current defined in ref. 10. Note that $D$ is independent of the electron beam radius, and hence so is $P_{\text {rad }}$ in Eq. (8.71) for the limit $R \ll 1$. From Eqs. (8.71) and (8.76) it follows that for small electron beam radius,

$$
P_{\text {rad }} \propto I_{0}^{3 / 2} .
$$

The average energy lost by an electron from start-up to saturation is determined from ${ }^{1}$

$$
\left\langle\sigma_{j}^{\prime}\right\rangle=\left\langle\frac{\Delta \gamma_{j}}{\rho \gamma}\right\rangle=-\nu .
$$

Usirg Eqs. (8.63) and (8.77) we find

$$
\left\langle\frac{\Delta \gamma_{j}}{\gamma}\right\rangle=-\nu \rho \approx-\frac{\bar{c}}{4} D
$$


consistent with Eq. (8.71). The energy spread at saturation is determined from

$$
\left.<\phi_{j}^{2}\right\rangle=\left\langle\left[\frac{\Delta \gamma_{j}}{\rho \gamma}-\left\langle\frac{\Delta \gamma_{j}}{\rho \gamma}\right\rangle\right]^{2}\right\rangle=2 \bar{c} P_{0},
$$

where the second equality follows from Eqs. (8.22) and (8.64). Now using Eqs. (8.62) and (8.76) we obtain for $R \ll 1$,

$$
\left[\left\langle\left(\frac{\Delta \gamma}{\gamma}-\left\langle\frac{\Delta \gamma}{\gamma}\right\rangle\right)^{2}\right\rangle\right]^{1 / 2} \approx \bar{c} D \sqrt{L} .
$$

The size of the radiation mode is seen from Eq. (8.27) to be given by

$$
r_{E M}=\frac{1}{\sqrt{\nu}}
$$

Introducing the actual size $\left(r_{d}\right)_{E M}$ in dimensioned units, we observe that

$$
r_{E M}^{2}=4 \rho k_{s} k_{w}\left(r_{d}\right)_{E M}^{2}=\frac{1}{\nu} \approx \frac{2}{\bar{c} R},
$$

where we used Eq. (8.63). Hence,

$$
\left(r_{d}\right)_{E M} \approx \frac{1}{\sqrt{c D k_{w} k}}
$$

Note that the size of the radiation beam given in Eq. (8.83) is independent of the electron beam radius in the small electron beam size limit.

Let us conclude this discussion of the small electron beam radius limit with a brief consideration of the dipole oscillation mode described by Eqs. (8.44)-(8.54). In the limit $R \rightarrow 0$, we observe that $p, q, \alpha$ each approach zero, but $\beta$ may remain finite. This is true because it turns out that $\lambda$, as defined in Eq. (8.42) is negative and approaches zero slowly, as the reciprocal of a logarithm. We find

$$
\begin{gathered}
\alpha^{2} \approx \nu R^{2}, \beta^{2} \approx 2|\lambda| R^{2}, \alpha^{2}+q^{2} \approx \Omega R^{2}, \alpha^{2}-p^{2} \approx-\Omega R^{2}, \\
\hat{K}^{-1} \approx \ln \frac{p}{2}+\gamma_{E}, \hat{H}^{-1} \approx \ln \frac{q}{2}+\gamma_{E}+\frac{i \pi}{2}, \hat{I} \approx \alpha^{2} / 2 .
\end{gathered}
$$

It follows that in the limit $R \rightarrow 0$, the dispersion relation of Eq. (8.52) becomes

$$
\frac{1}{\hat{K}}+\frac{1}{\hat{H}}=\frac{2}{\hat{J}}
$$


yielding

$$
\ln (p q / 4)+2 \gamma_{E}+i \pi / 2=\frac{J_{0}(\beta)}{\beta J_{0}^{\prime}(\beta)} .
$$

Since the left hand side of Eq. (8.84) diverges as $R \rightarrow 0$, one solution corresponds to $\beta=\beta_{1}=3.83$, the first zero of $J_{1}(\beta)$. In this case

$$
\beta^{2}=2|\lambda| R^{2}=\frac{2 \bar{c} R^{2}}{2 \bar{c} P_{0}-\Omega^{2}}=(3.83)^{2} .
$$

Using Eq. (8.62) for $P_{0}$, we find

$$
\Omega^{2}=\bar{c}^{2} R^{2}\left(L-2 / \beta^{2}\right)=\bar{c}^{2} R^{2}(L-0.14),
$$

where the logarithmic factor $L$ was defined preceding Eq. (8.62).

Let us now solve Eq. (8.84) more accurately by expanding about $\beta=\beta_{1}$. We find

$$
\beta-\beta_{1}=\frac{1}{\beta_{1}}\left[\frac{-1}{L_{1}}+\frac{i \pi}{2 L_{1}^{2}}\right],
$$

where $L_{1}=\ell n(p q / 4)+2 \gamma_{E}$. As a consequence it is seen from Eq. (8.86) that $\Omega$ has a positive imaginary part of order $R /(\ln R)^{5 / 2}$.

There are other solutions of the dispersion relation, Eq. (8.52), but we shall not consider them here. To understand the coherent dipole mode oscillation better, it seems necessary to compare the analytic results with simulations.

\section{Summary}

Starting with the equations of Bonifacio et al. ${ }^{1}$ we explore the behavior of an unbunched electron beam interacting with a low amplitude of radiation. The level of radiation grows exponentially causing the electrons to bunch. When the bunching saturates, the radiation amplitude starts to perform what appears to be oscillations in the saturation region. By making an assumption regarding the phase of the radiation which is suggested by the simulations, we predict the general features of the electron bunching, average radiation amplitude and the frequency and magnitude of the oscillations of the radiation amplitude which are in good agreement with the results of detailed simulations of the starting equations.

We consider three quite different equilibrium electron phase space distributions, and find the surprising result that the equilibrium radiation amplitude $P_{0} \cong 0.81$, 
independent of the distribution. In addition, from simulations of start-up from an initially unbunched electron beam and a small radiation amplitude, we find that the saturated state is described by oscillations about a distribution similar to the Boltzmann distribution. This observation might provide the starting point for a future investigation.

In the case of the KV-distribution, we use the Vlasov equation to study the stability of the oscillations about the equilibrium state. For a small bunch ( $\theta_{0}$ small) the oscillations are stable. However, for a large bunch $\left(\theta_{0}=81^{\circ}\right)$ required by the invariants in the case of start-up from an unbunched electron beam and a low radiation amplitude, the coherent dipole oscillation mode is found to be unstable. We believe that the moderate amplitude oscillations, observed in the simulation from start-up with an unbunched electron beam and a low radiation amplitude, corrrespond to the stabilization of the dipole mode at large oscillation amplitude. The real part of the coherent dipole mode frequency is in good agreement with the oscillation frequency observed in the saturated state. There are some instabilities in the higher-order coherent oscillation modes, but these are not seen in the simulations since they are not stimulated by our starting conditions.

Finally, we extend the analysis to the two-dimensional case of an electron beam with finite radial extent. We find an equilibrium guided solution and oscillations about it. There are two types of oscillation modes, one guided and one corresponding to radiation propagating to $r=\infty$. The escape of radiation from the electron beam leads to a damping of the oscillations. Explicit results are obtained in the limits of large and small electron beam radius.

In saturation, the output power has contributions from the equilibrium ( $z$-independent) mode and the coherent dipole oscillation (z-dependent) mode. In the case of start-up from an unbunched electron beam and a low initial radiation level, the saturated power $P_{\text {rad }}$ in the equilibrium mode is given by [Eq. (8.71)]:

$$
P_{\text {rad }} / P_{e}=\nu \rho,
$$

where $P_{e}$ is the power in the electron beam. In general $\nu$ can be determined by solving Eqs. (8.21), (8.22), (8.28) and (8.30). In the limits of large and small electron beam radius:

$$
\nu=0.70, P_{\text {rad }} / P_{e}=0.35 D / R,(R \gg 1)
$$


and

$$
\nu=0.32 R, P_{\text {rad }} / P_{e}=0.16 D,(R \ll 1)
$$

where $D$ [Eq. (8.75)] depends on the electron beam current, but is independent of the radius, and the scaled electron beam radius $R$ is related to the dimensioned radius $R_{d}$ by Eq. (8.74).

$$
R^{2}=4 \rho k_{w} k_{s} R_{d}^{2}
$$

In the limit of small radius, we find that the equilibrium mode power $P_{\text {rad }}$ increases with current $I_{0}$ according to

$$
P_{\text {rad }} / P_{e} \propto I_{0}^{3 / 2}
$$

a current dependence intermediate between the incoherent $\left(I_{0}\right)$ and fully coherent $\left(I_{0}^{2}\right)$ limits.

Comparison of the two-dimensional analysis with computer simulations is an interesting subject for future investigation. 


\section{A Dispersion Relation for Small Oscillations}

The behavior of small oscillations about the equilibrium solution is governed by the Vlasov equation

$$
\frac{\partial f}{\partial \tau}+\frac{\partial H}{\partial I} \frac{\partial f}{\partial \psi}-\frac{\partial H}{\partial \psi} \frac{\partial f}{\partial I}=0
$$

Using the Hamiltonian in Eqs. (5.6) and (5.7) and linearizing the distribution function in the form

$$
f(\psi, I ; \tau)=f_{0}(I)+f_{1}(\psi, I ; \tau)
$$

we find in the linear approximation

$$
\frac{\partial f_{1}}{\partial \tau}+\omega \frac{\partial f_{1}}{\partial \psi}=\frac{\partial V}{\partial \psi} \frac{\partial f_{0}}{\partial I}
$$

Using Eqs. (5.7), (5.15), (5.16), we find

$$
\frac{\partial f_{1}}{\partial \tau}+\omega \frac{\partial f_{1}}{\partial \psi}=-2 P_{\circ} f_{\circ}^{\prime}(I) \sum_{n=0}^{\infty}\left[2 n Q A_{n} \sin 2 n \psi+(2 n+1) R B_{n} \cos (2 n+1) \psi\right],
$$

whose solution is

$$
\begin{aligned}
f_{1}(\psi, I ; \tau) & =-2 P_{\circ} f_{0}^{\prime}(I) \sum_{n=0}^{\infty}\left[C_{n} \cos 2 n \psi+D_{n} \sin 2 n \psi+E_{n} \cos (2 n+1) \psi\right. \\
& \left.+F_{n} \sin (2 n+1) \psi\right]
\end{aligned}
$$

where Eq. (A.4) requires that

$$
\begin{gathered}
C_{n}^{\prime}+2 n \omega D_{n}=0, D_{n}^{\prime}-2 n \omega C_{n}=2 n \tilde{P} A_{n}, \\
E_{n}^{\prime}+(2 n+1) \omega F_{n}=(2 n+1) Q B_{n}, F_{n}^{\prime}-(2 n+1) \omega E_{n}=0 .
\end{gathered}
$$

We now assume an oscillation mode for $\tilde{P}, Q, C_{n}, D_{n}, E_{n}, F_{n}$ of the form $\exp (-i \Omega \tau)$ and find

$$
\begin{gathered}
\frac{2 n \omega}{\Omega} D_{n}=C_{n}=-\frac{(2 n)^{2} \omega \tilde{P} A_{n}}{(2 n)^{2} \omega^{2}-\Omega^{2}}, \\
\frac{-(2 n+1) \omega}{i \Omega} E_{n}=F_{n}=\frac{(2 n+1)^{2} \omega Q B_{n}}{(2 n+1)^{2} \omega^{2}-\Omega_{2}} .
\end{gathered}
$$

We must now relate $\tilde{P}$ and $Q$ to the perturbed distribution by using Eqs. (5.4) and (5.5). After performing the integration over $\psi$, and integrating by parts over $I$, we obtain

$$
-i \Omega Q+\left[\nu-\sum_{n=0}^{\infty} S_{n}(\Omega)\right] \tilde{P}=0
$$




$$
i \Omega \tilde{P}+\left[\nu-\sum_{n=0}^{\infty} T_{n}(\Omega)\right] Q=0
$$

where

$$
\begin{gathered}
S_{n}(\Omega)=2 \pi \int d I f_{\circ}(I) \frac{d}{d I}\left[\frac{(2 n)^{2} \omega A_{n}^{2}}{(2 n)^{2} \omega^{2}-\Omega^{2}}\right], \\
T_{n}(\Omega)=2 \pi \int d I f_{0}(I) \frac{d}{d I}\left[\frac{(2 n+1)^{2} \omega B_{n}^{2}}{(2 n+1)^{2} \omega^{2}-\Omega^{2}}\right] .
\end{gathered}
$$

Finally, Eqs. (A.10) and (A.11) yield the dispersion relation for the oscillation modes:

$$
\left[\nu-\sum_{n=1}^{\infty} S_{n}(\Omega)\right]\left[\nu-\sum_{n=0}^{\infty} T_{n}(\Omega)\right]=\Omega^{2}
$$

whose solution determines the modes of oscillation. Since $A_{n}$ and $B_{n}$, defined in Eqs. (5.16) and (5.17), are real, stability requires that all solutions for $\Omega$ in Eq. (5.19) be real.

\section{References}

1. R. Bonifacio, F. Casagrande and L. DeSalvo Souza, Phys. Rev. A $\underline{33}, 2836$ (1986).

2. B. Lane and R.C. Davidson, Phys. Rev. A27, 2008 (1983).

3. R.C. Davidson and J.S. Wurtele, Phys. Fluids $\underline{30}, 557$ (1987).

4. W.M. Sharp and S.S. Yu, Nucl. Instrurn. Meth. A 272,397 (1988).

5. W.M. Sharp and S.S. Yu, Phys. Fluids B 2,581 (1990).

6. See e.g. R. Bonifacio, C. Pellegrini and L.M. Narducci, Opt. Commun. $\underline{50}, 373$ (1984).

7. For a review of FEL theory and simulations, see e.g. J.B. Murphy and C. Pellegrini in Laser Handbook, Vol. 6, eds. W.B. Colson, C. Pellegrini and A. Renieri, North-Holland Pub. Co., Amsterdam, 1990, p. 9.

8. These equations are equivalent to those considered in ref. 5 , in the case when the radiation field depends on the axial coordinate $z$, but is independent of time $t$.

9. See, for example Abramowitz and Stegun, Handbook of Mathematical Functions, Dover Publications, NY, 1965. 
10. L.H. Yu, S. Krinsky, and R.L. Gluckstern, Phys. Rev. Lett. 64, 3011 (1990).

11. The scaled electron beam radius $R$ is denoted $\tilde{a}$ in ref. 10 and in S. Krinsky and L.H. Yu, Phys. Rev. A 35,3406 (1987). $R^{2}$ is proportional to the ratio of the Rayleigh range to the one-dimensional gain length. 


\section{FIGURE CAPTIONS}

Fig. 1.1. Evolution of radiation field amplitude $|A|$ with $\tau$.

Fig. 1.2. Phase of the radiation field as function of $\tau$.

Fig. 3.1. Phase space distribution of the electrons, which were initially monoenergetic with uniform phase distribution, in the saturation regime $\tau \sim 9$.

Fig. 3.2. Evolution of $P$ and $Q$ with $\tau$.

Fig. 3.3. Phase space distribution in the $\beta_{j}^{\prime}, \beta_{j}$ space at $\tau=10,15,20,25,30,35$.

Fig. 3.4. The three distributions, $\mathrm{KV},\left(H_{0}-H\right)^{1 / 2}$ and Boltzmann plotted as functions of $H$ defined in Eq. (3.11).

Fig. 3.5. Electron distributions obtained from simulation for $\tau=10,20,30,40$ plotted as functions of $H$.

Fig. 4.1. Plot of $P^{\prime}(\tau)$ vs $P(\tau)$.

Fig. 6.1. Electron phase space distribution corresponding to $\mathrm{KV}$ equilibrium solution for small pendulum angle $\theta_{0}=3^{\circ}$.

Fig. 6.2. Equilibrium value of $P(\tau)=P_{0}$ for $\mathrm{KV}$ equilibrium solution with $\theta_{0}=3^{\circ}$.

Fig. 6.3. Oscillation of $|A(\tau)|$ about $\mathrm{KV}$ equilibrium solution with $\theta_{0}=3^{\circ}$, when simulation started with $P(0) / P_{0}=0.99$.

Fig. 6.4. Oscillation of $|A(\tau)|$ about $\mathrm{KV}$ equilibrium sulution with $\theta_{0}=30^{\circ}$, when simulation started with $P(0) / P_{0}=0.99$.

Fig. 6.5. Oscillation of $|A(\tau)|$ about $\mathrm{KV}$ equilibrium solution with $\theta_{0}=55^{\circ}$, when simulation started with $P(0) / P_{0}=0.99$. Oscillation unstable in this case.

Fig. 6.6. Oscillation of $|A(\tau)|$ about $\mathrm{KV}$ equilibrium solution with $\theta_{0}=80^{\circ}$, when simulation started with $P(0) / P_{0}=0.99$. Oscillation unstable in this case.

Fig. 6.7. Coherent frequency $\Omega$ obtained from numerical solution of the dispersion relation of Eq. (5.19), where only dipole term $T_{0}$ has been included. Instability predicted for $\theta_{0}>50^{\circ}$, consistent with results of Figs. 6.3-6.6. The dashed curve represents the coherent frequency found in the single harmonic model of Section 4.

Fig. 6.8. Coherent frequency $\Omega$ obtained from numerical solution of the dispersion relation of Eq. (5.19), including several additional terms. 
Fig. 7.1. Solution of Eqs. (7.2) and (7.3) for $P_{0}$ as a function of $\delta$, for $\epsilon^{2}=0$, is plotted as solid curve. In addition, results from simulation are also shown. 


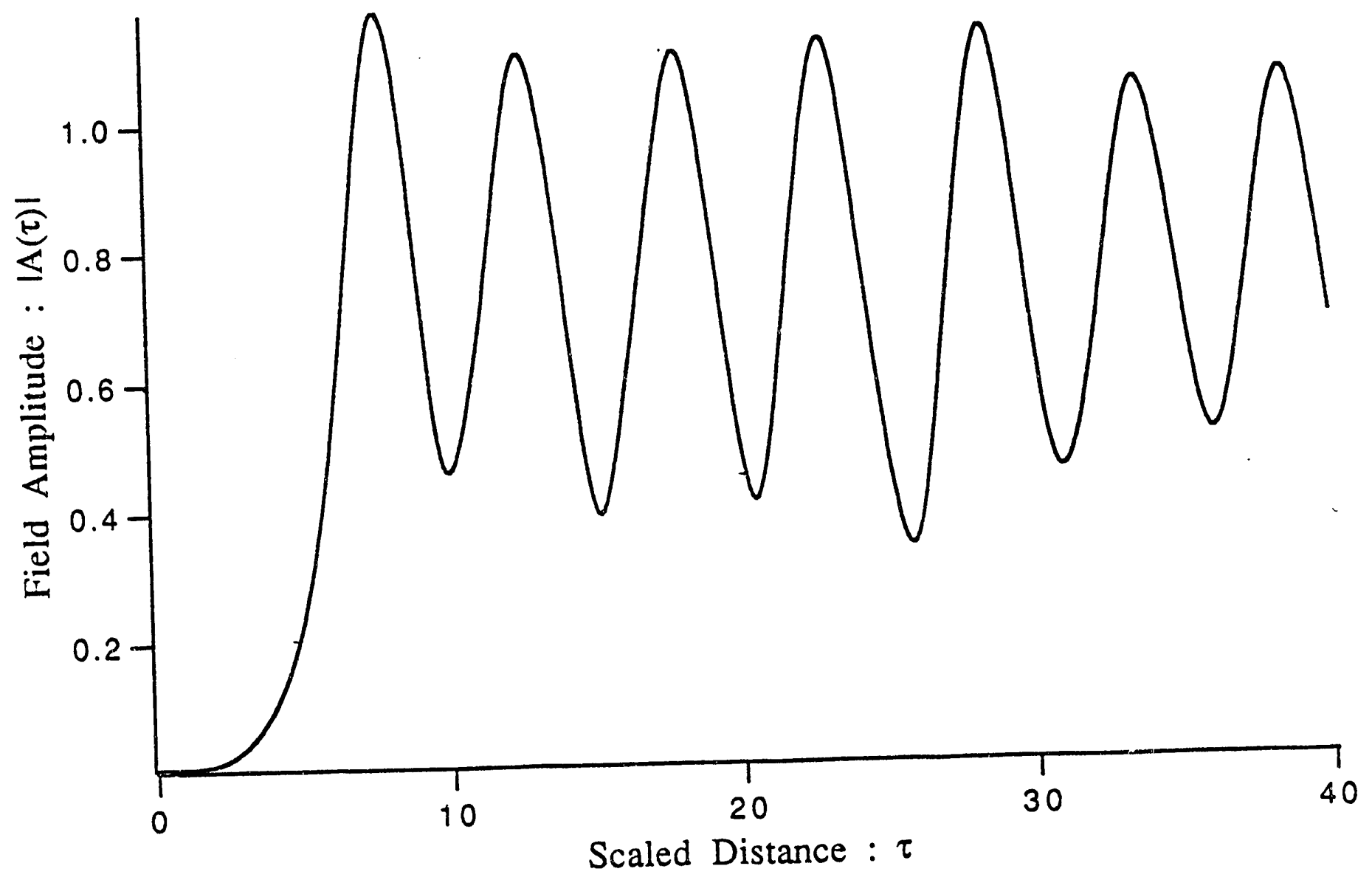

Fig.1.1 


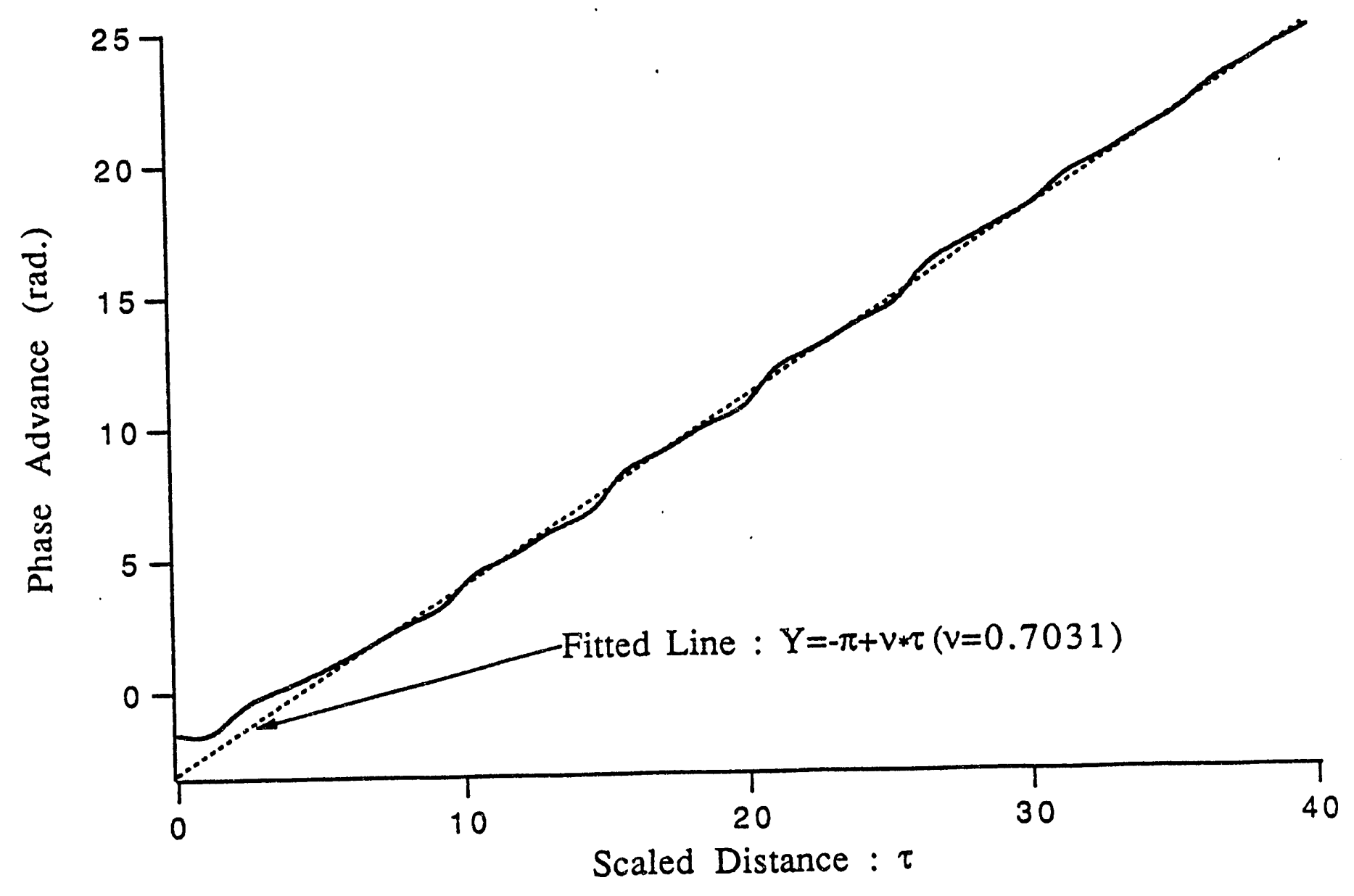

Fig.1.2 


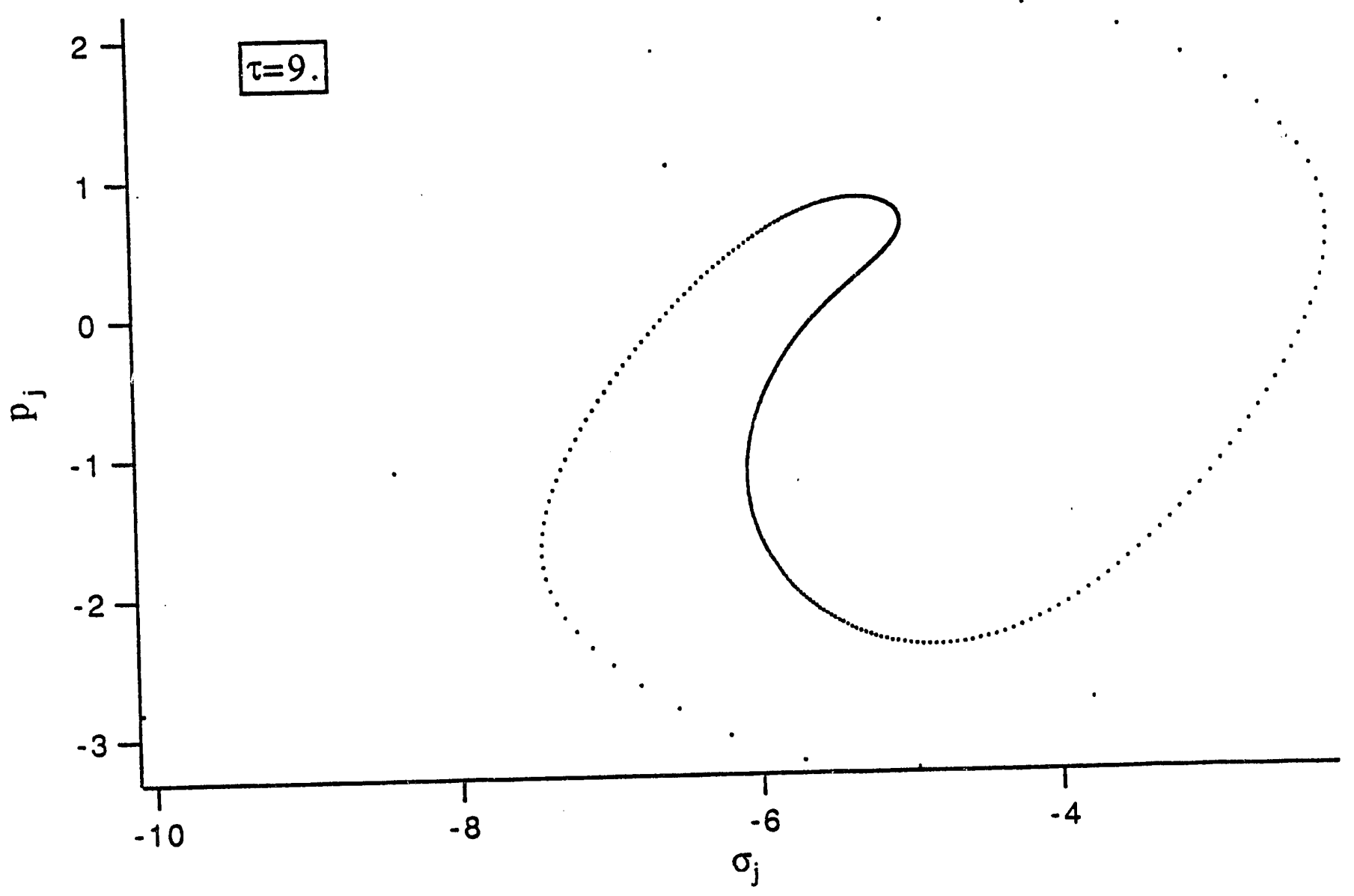

Fig.3.1 


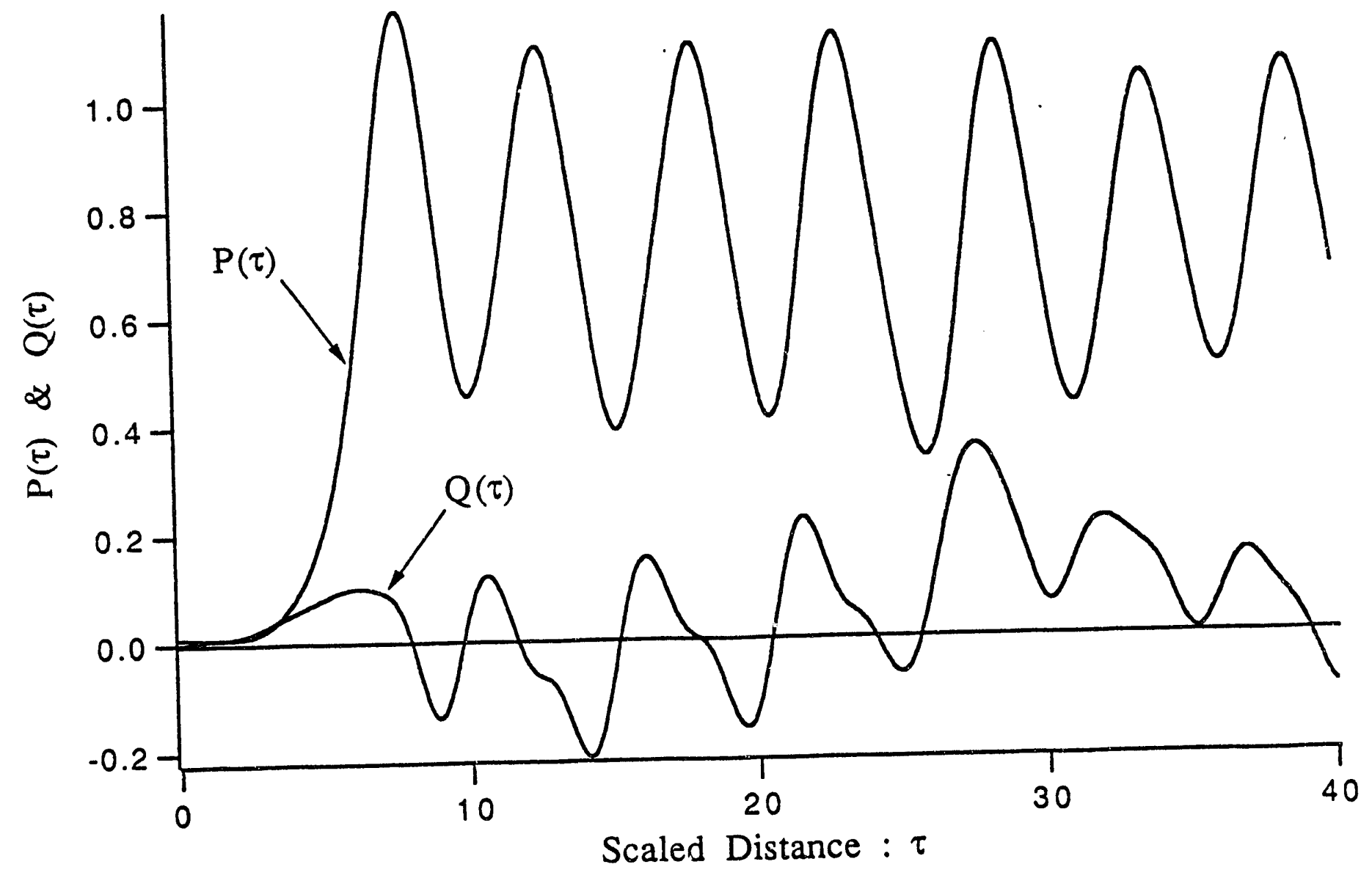

Fig.3.2 

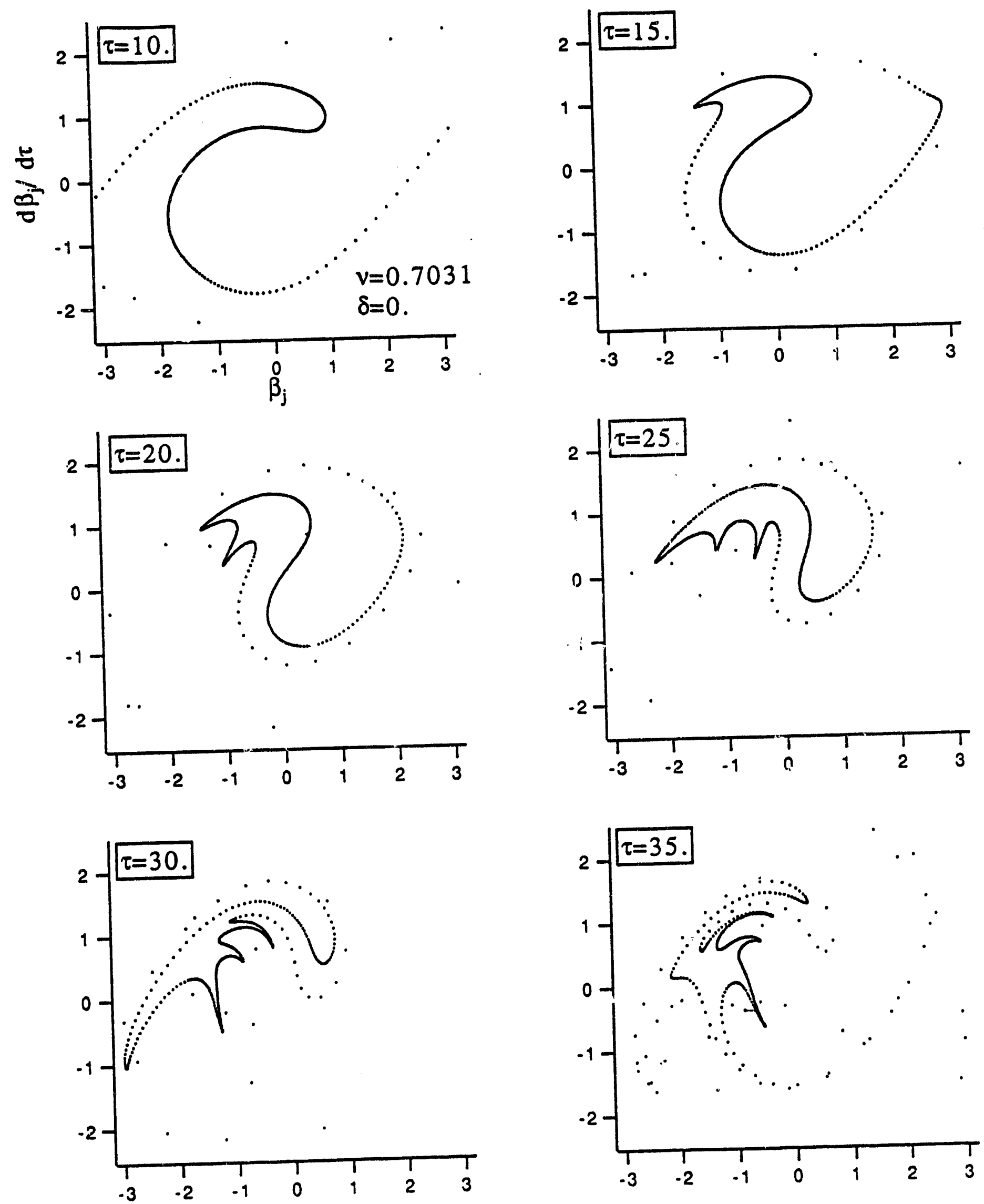

Fig.3.3 


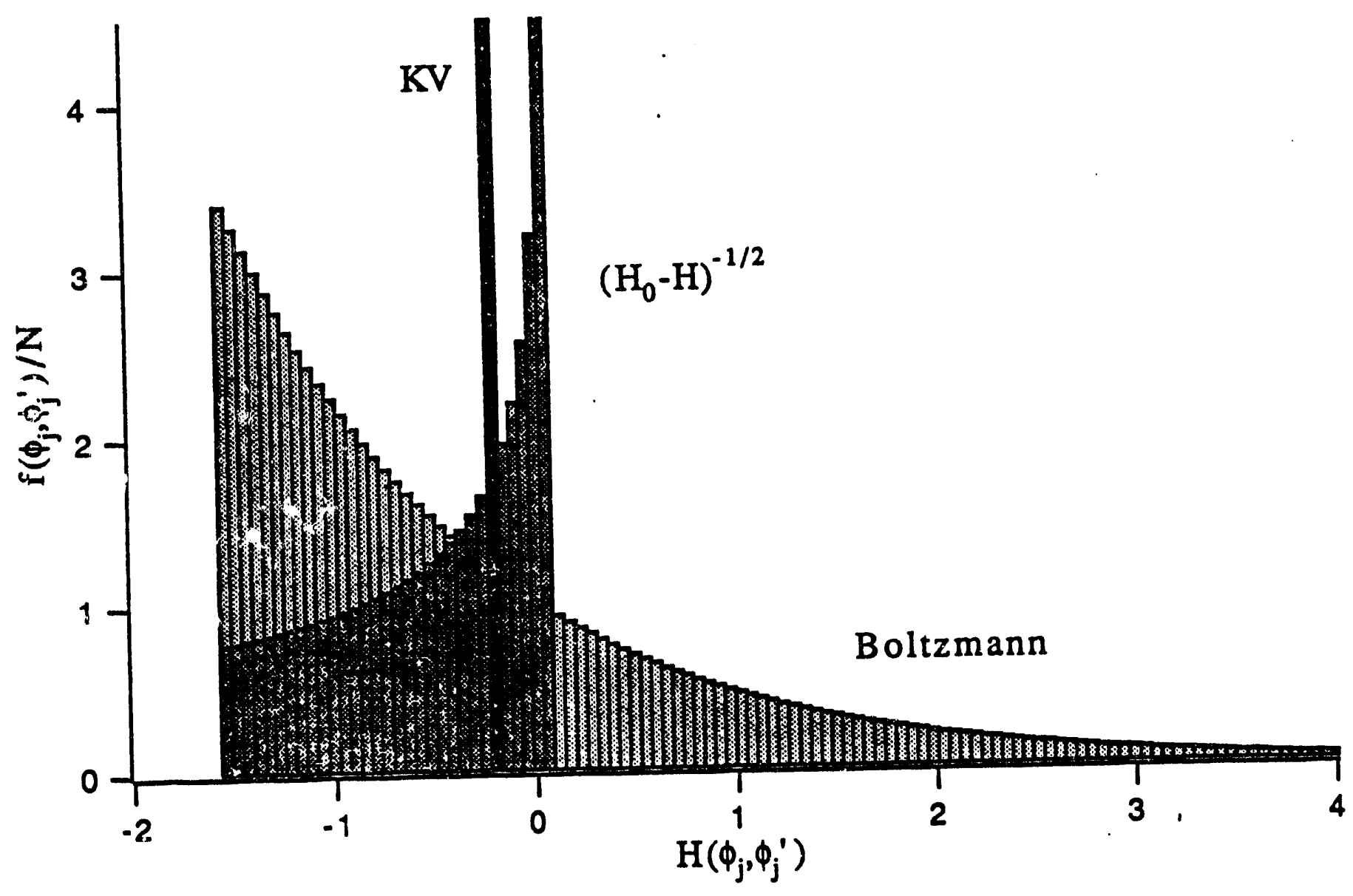

Fig.3.4 

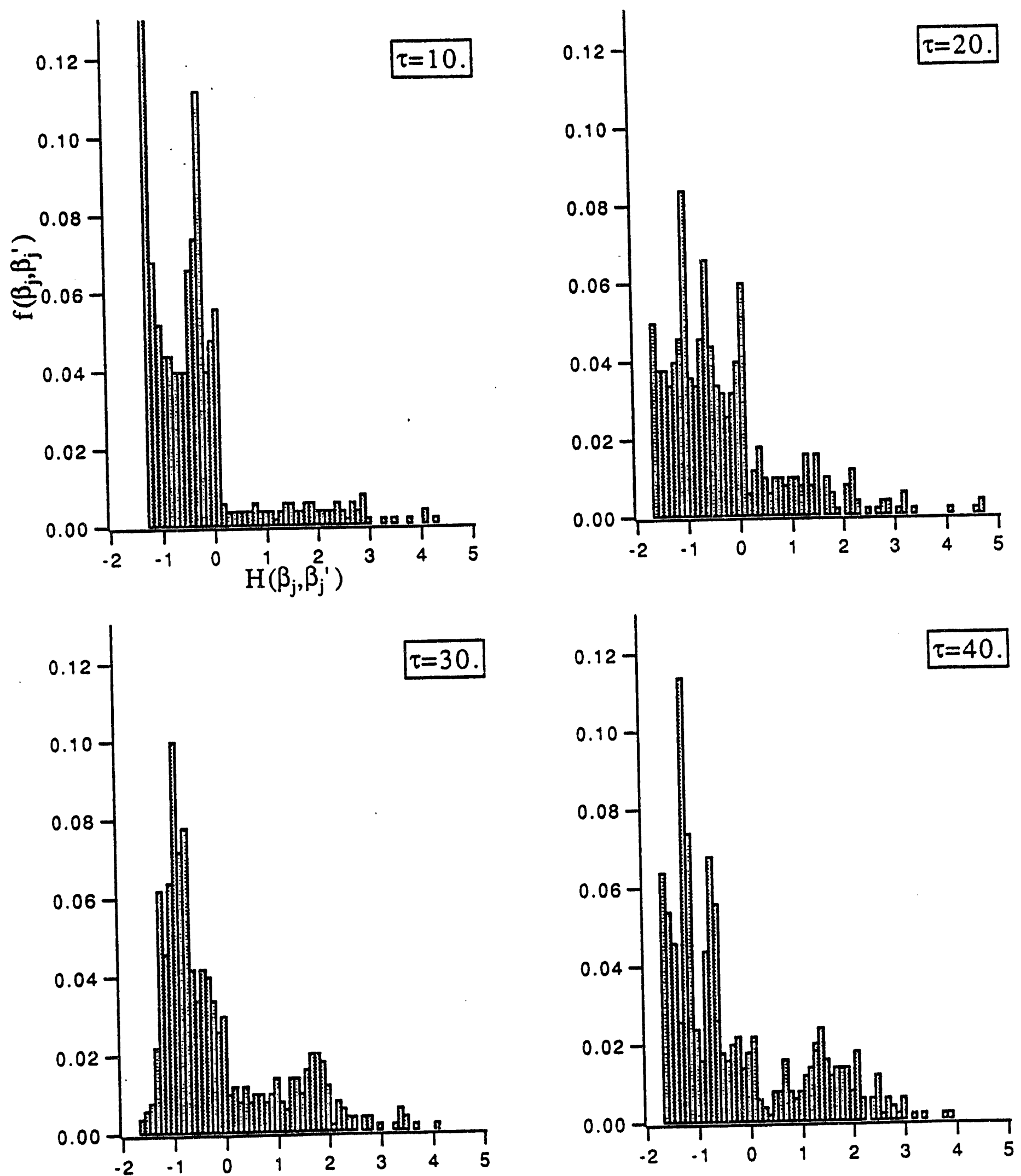

Fig.3.5 


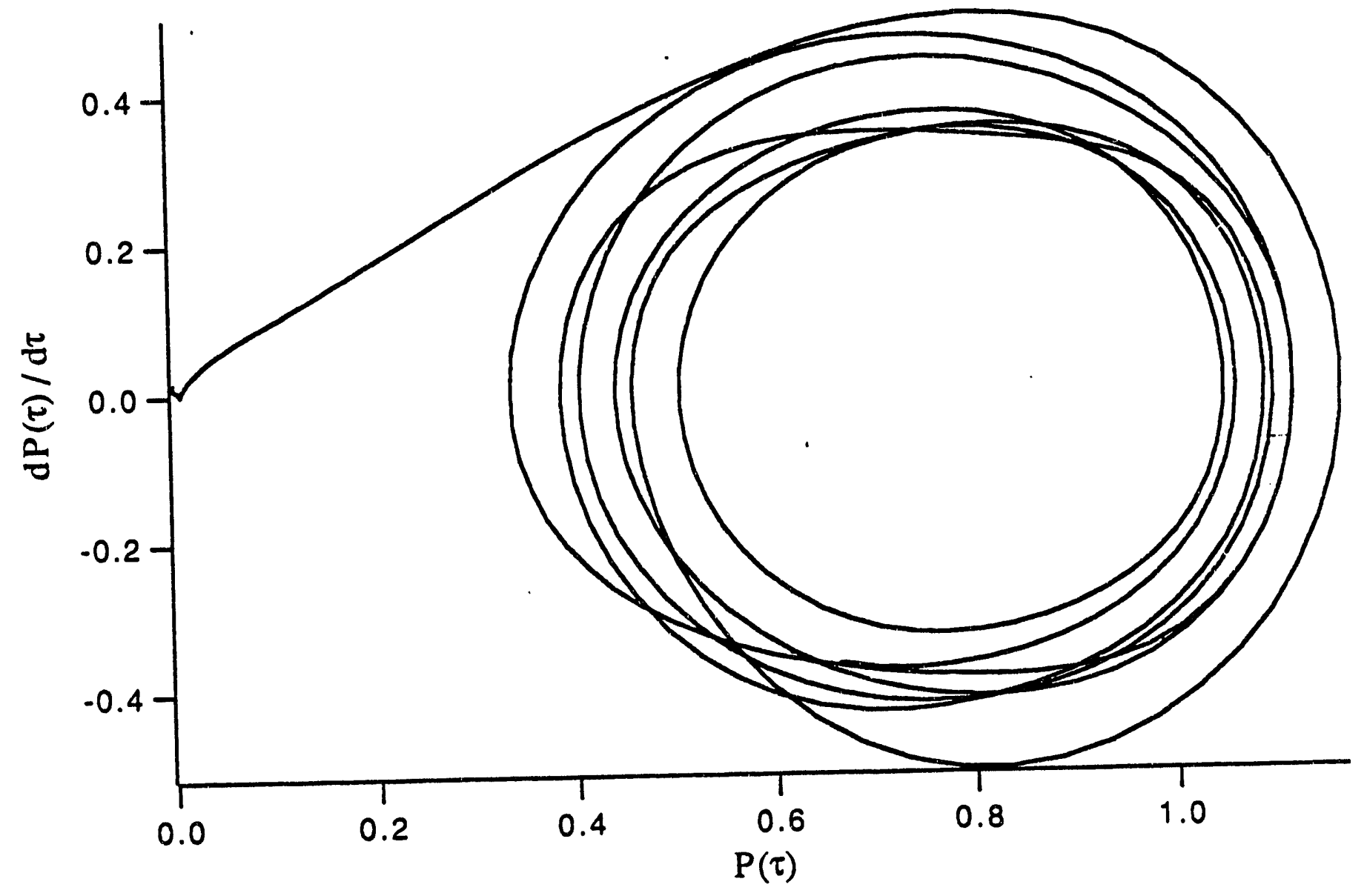

Fig.4.1 

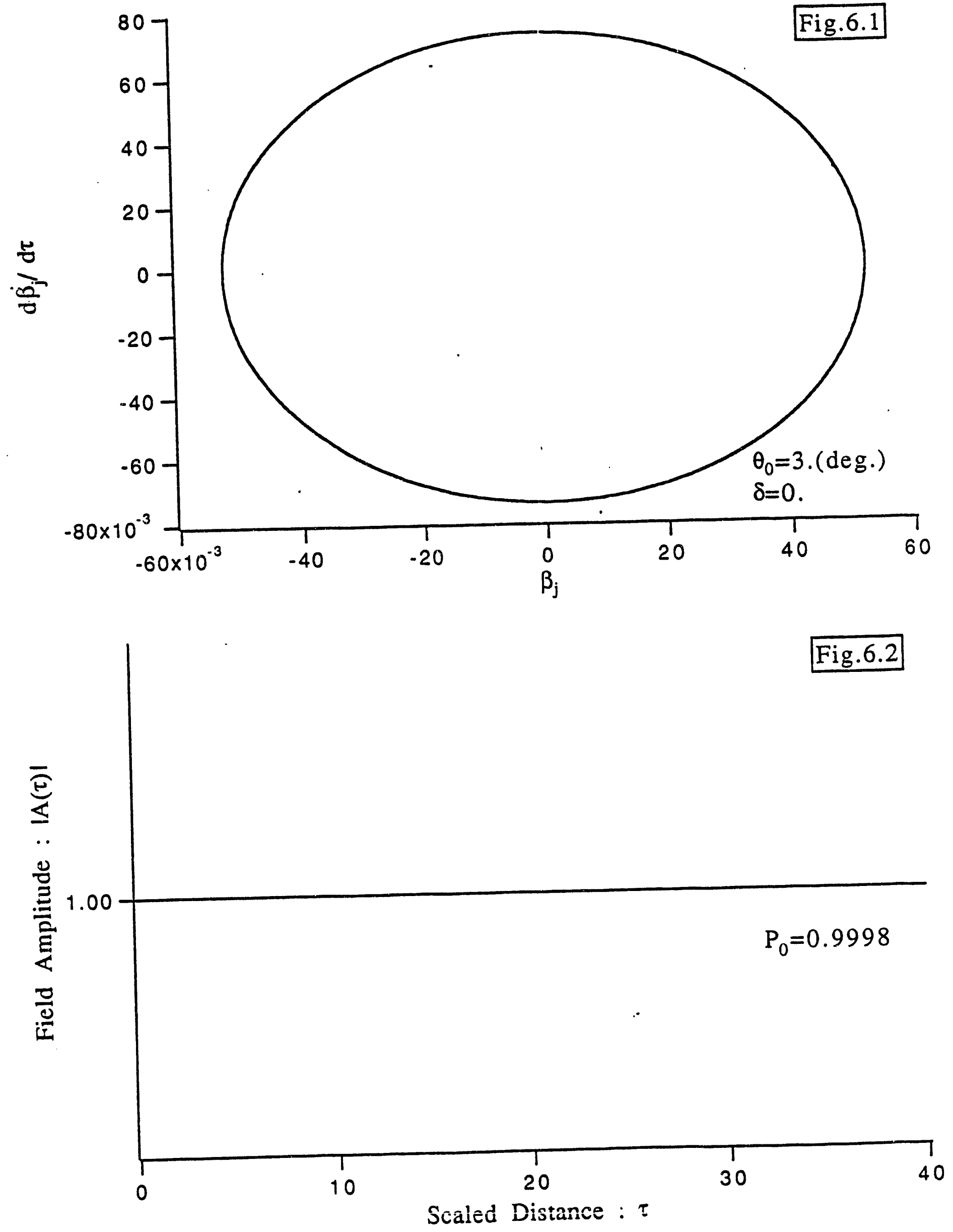


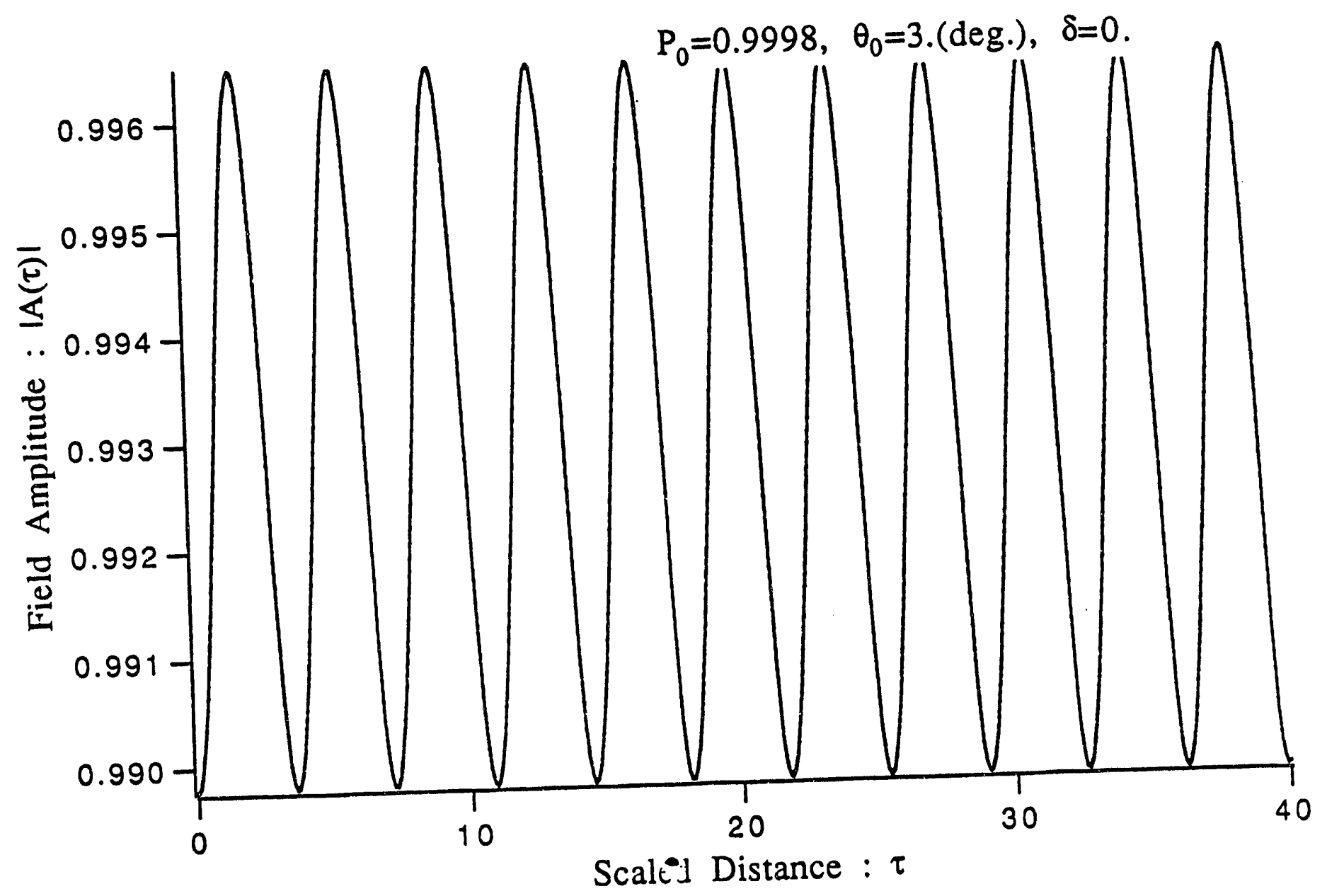

Fig.6.3 


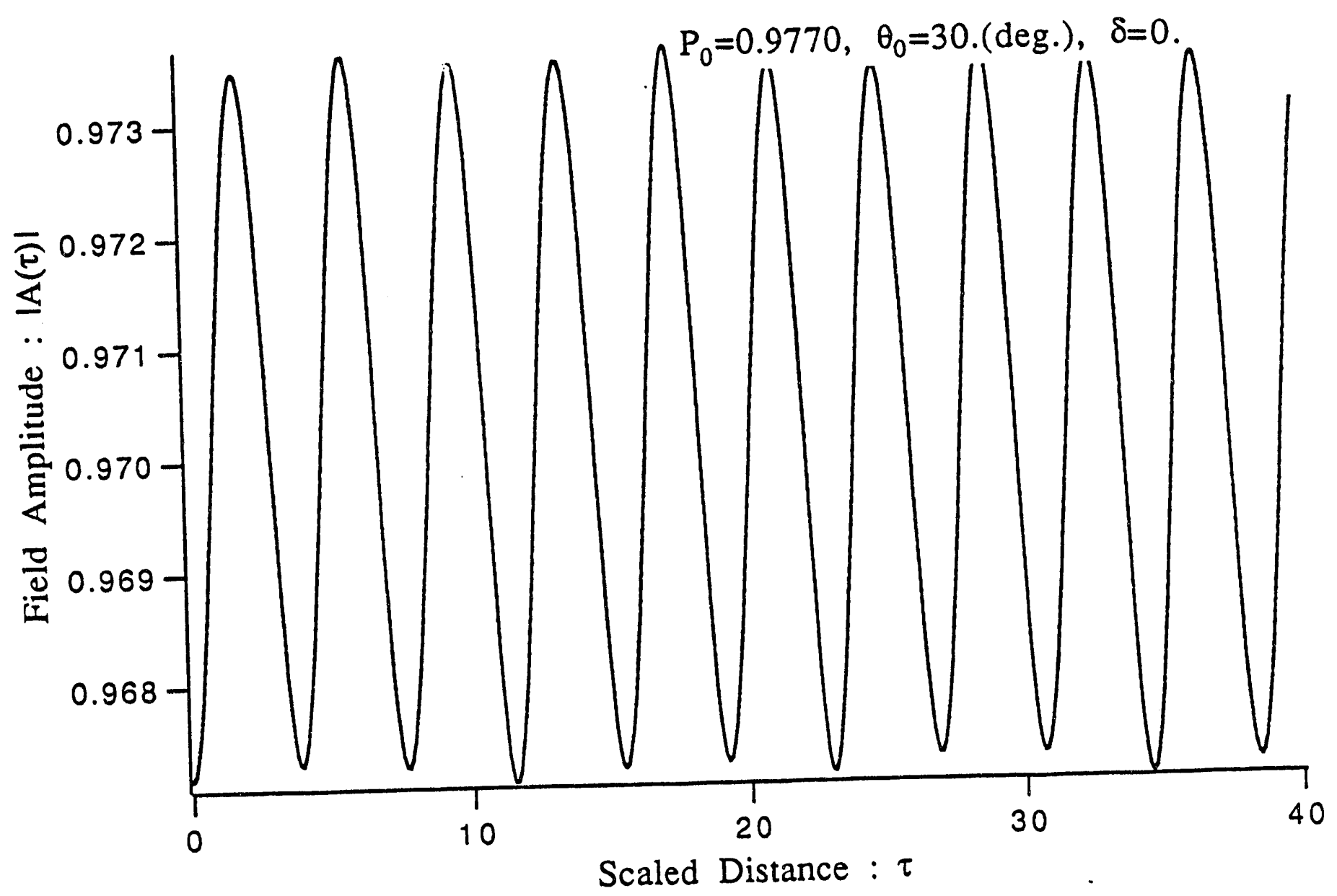

Fig.6.4 


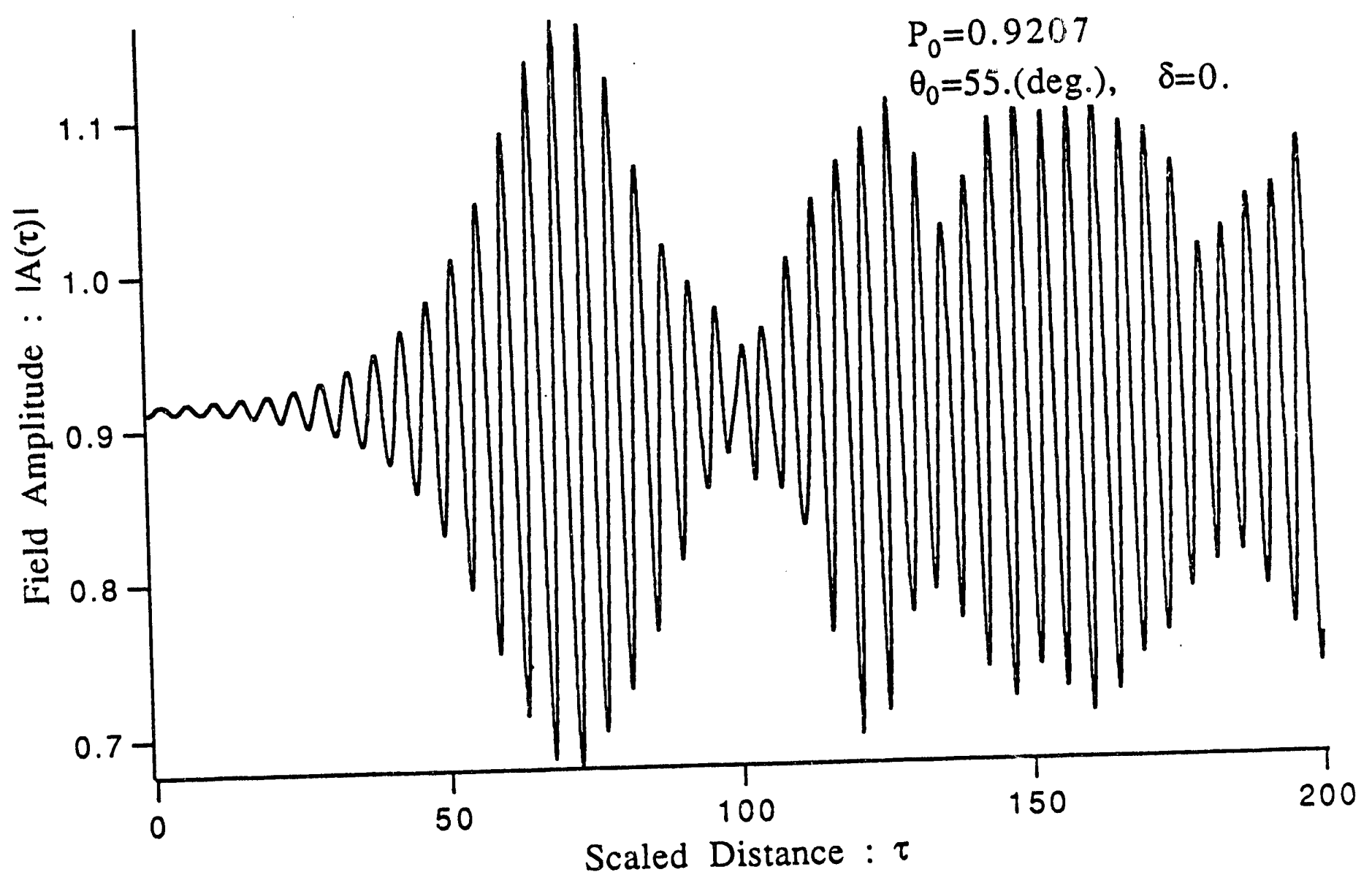

Fig.6.5 


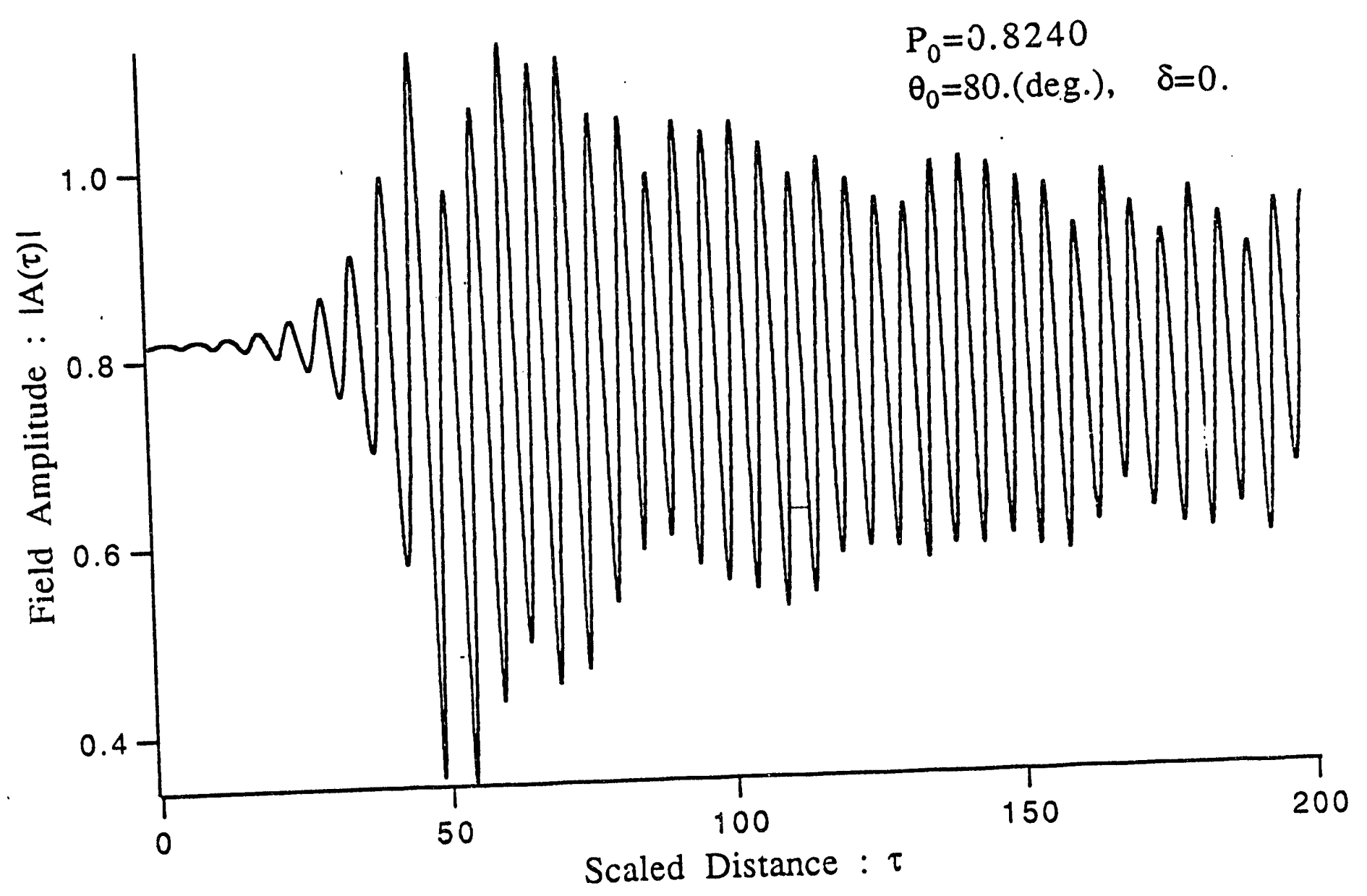

Fig.6.6 

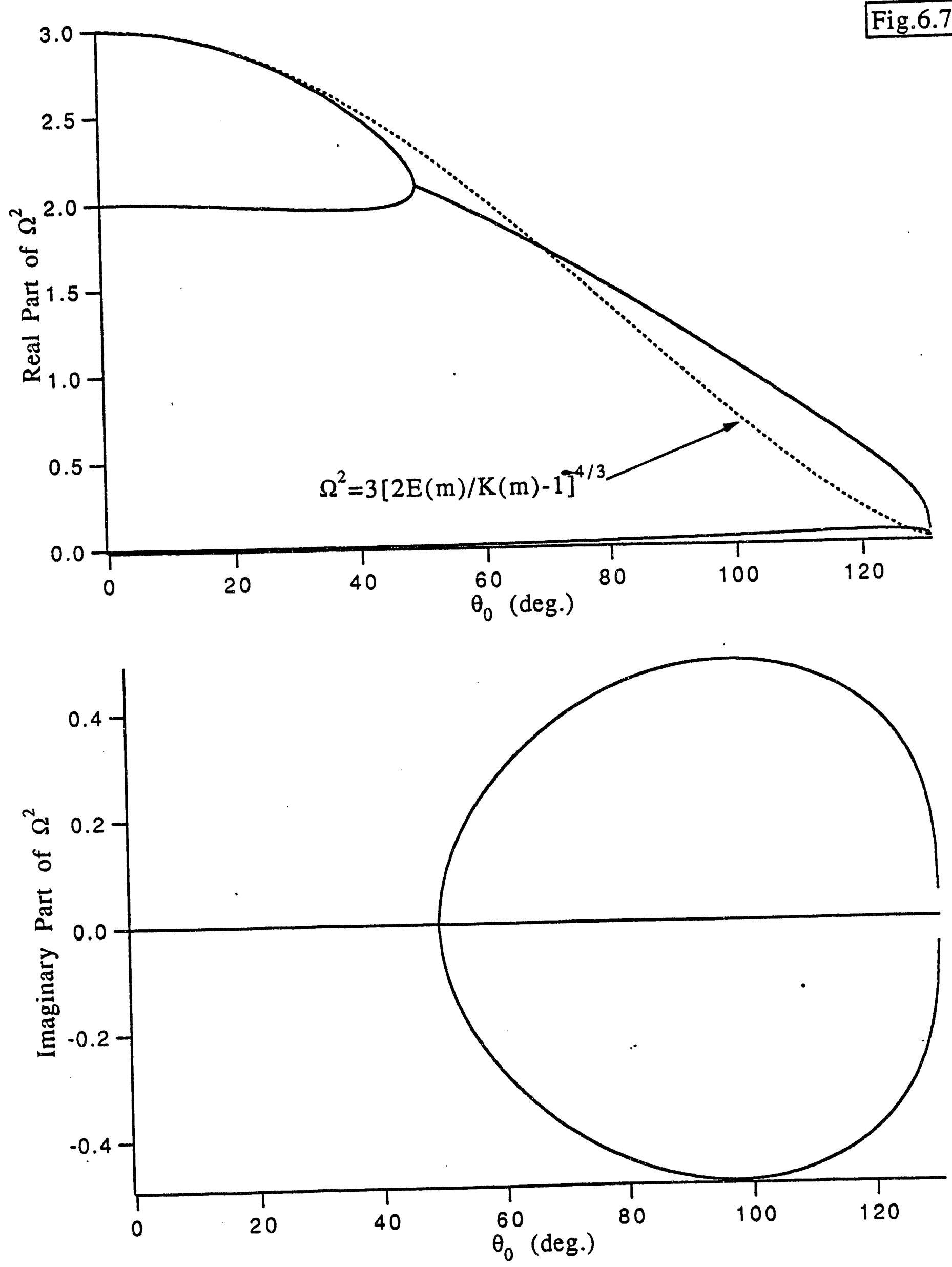

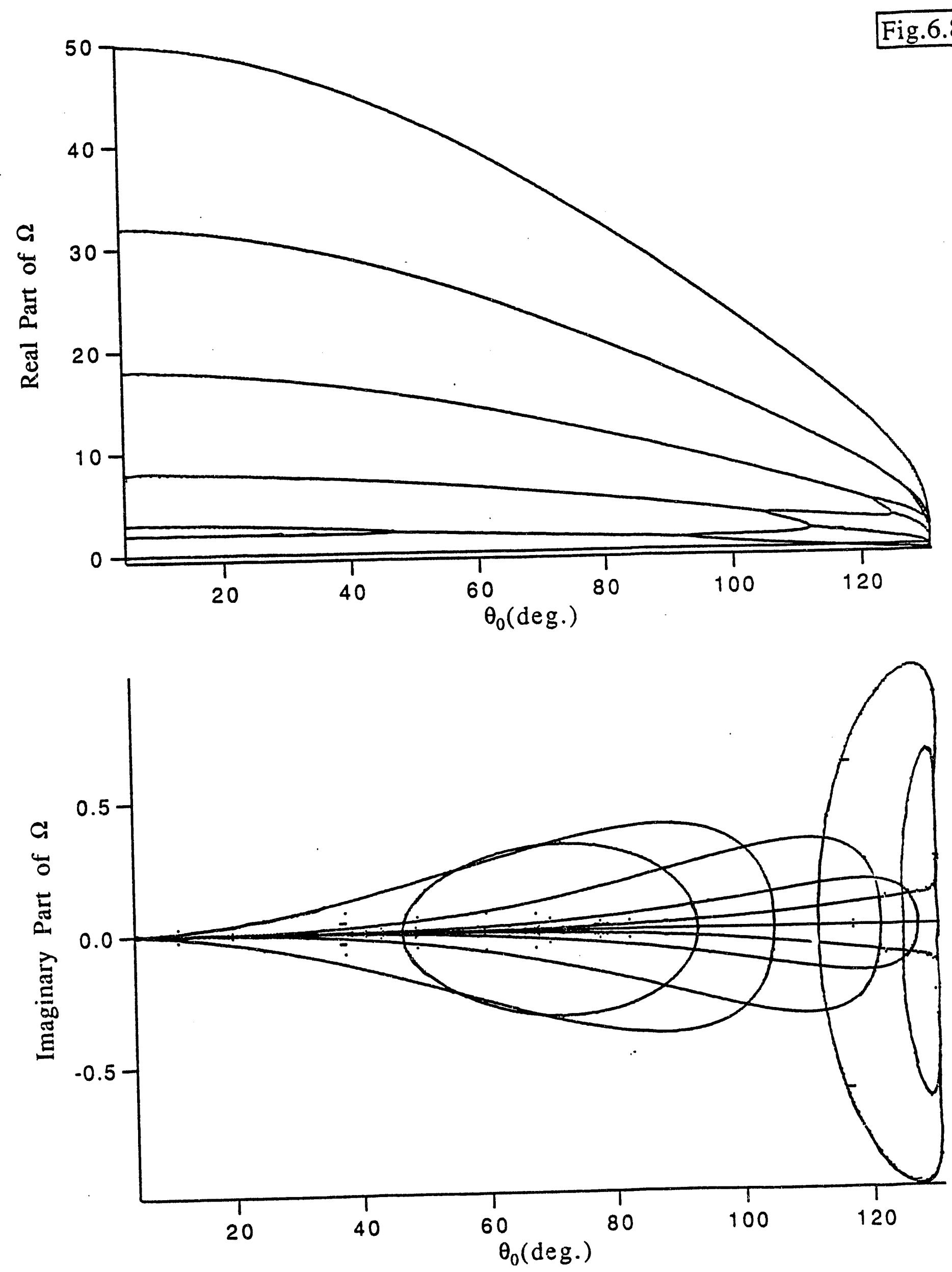


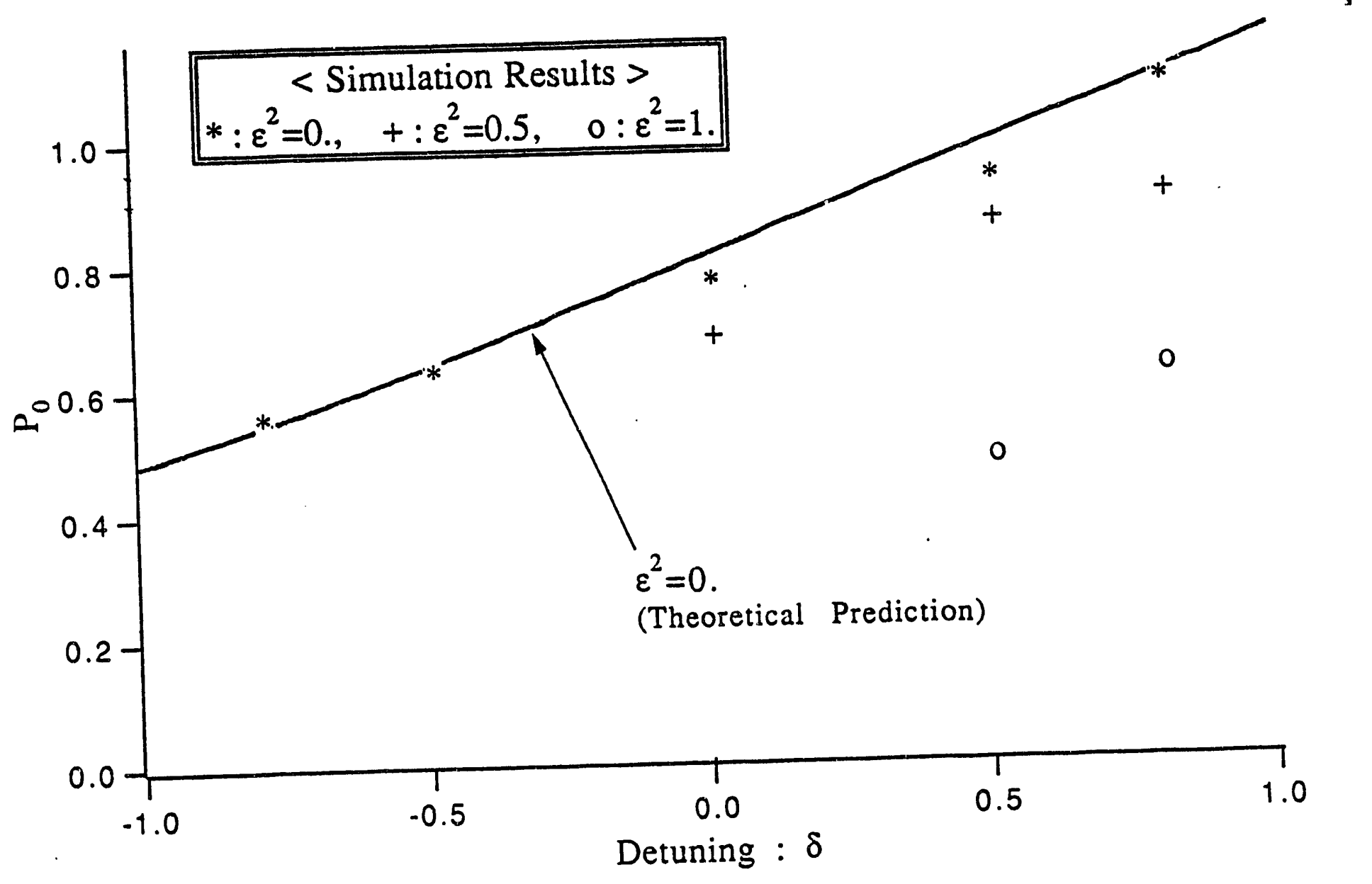

Fig.7.1 

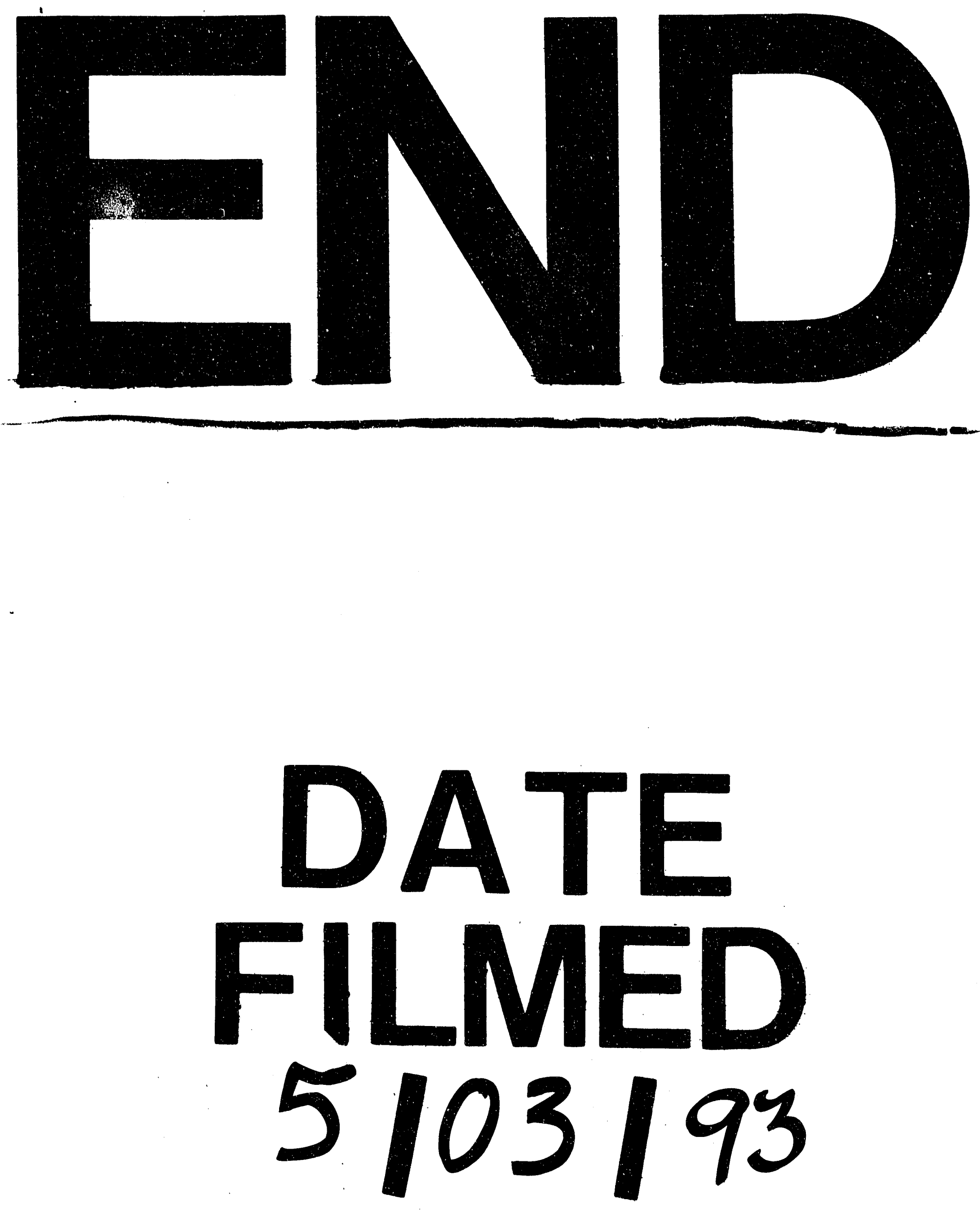
